\title{
1 Cortical thinning and altered functional brain coherence in survivors of
}

\section{childhood sarcoma}

3 Charlotte Sleurs ${ }^{1}$, Jeroen Blommaert ${ }^{1}$, Dafnis Batalle ${ }^{2,3}$, Marjolein Verly ${ }^{4}$, Stefan Sunaert ${ }^{5}$,

$4 \quad$ Ron Peeters ${ }^{5}$, Jurgen Lemiere ${ }^{6}$, Anne Uyttebroeck ${ }^{1,6}$, Sabine Deprez ${ }^{7}$

5

$6 \quad{ }^{1}$ Department of Oncology, KU Leuven, Leuven, Belgium

$7 \quad{ }^{2}$ Department of Forensic \& Neurodevelopmental Science, Institute of Psychiatry, Psychology

8 \& Neuroscience, King's College, London, United Kingdom

$9{ }^{3}$ Centre for the Developing Brain, School of Biomedical Engineering \& Imaging Sciences,

10 King's College London, United Kingdom

$11{ }^{4}$ Department of Neurosciences, ExpORL, KU Leuven, Belgium

$12{ }^{5}$ Department of Radiology, University Hospital Leuven, Belgium

$13{ }^{6}$ Department of Pediatric Hematology and Oncology, University Hospital Leuven, Belgium

$14{ }^{7}$ Department of Imaging and Pathology, KU Leuven, Leuven, Belgium

17 Corresponding author:

18 Charlotte Sleurs

19 UZ Leuven

20 Pediatric Hematology-Oncology

21 Herestraat 49

223000 Leuven

23 Tel: 0032/16343991

24 e-mail: charlotte.sleurs@kuleuven.be

25

26

27 Short running title: Cortical development in childhood sarcoma patients

28 Word count: 3952 


\section{Abstract}

High-dose chemotherapy is increasingly evidenced to be neurotoxic and result in long-term neurocognitive sequelae. However, research investigating grey matter alterations in childhood cancer patients remains limited. As childhood sarcoma patients receive high-dose chemotherapy, we aimed to investigate cortical brain alterations in adult survivors. We analyzed high-resolution structural (T1-weighted) MRI and resting-state functional MRI (rsfMRI), to derive structural and functional cortical information in survivors of childhood sarcoma, treated with high-dose intravenous chemotherapy $(n=33)$. These scans were compared to age- and gender- matched controls $(n=34)$. Cortical volume and thickness were investigated using voxelbased morphometry and vertex-wise surface-based morphometry. Brain regions showing significant group differences in volume or thickness were implemented as seeds of interest to estimate their resting state co-activity with other areas (i.e. functional coherence). We explored whether structural measures were associated with potential risk factors, such as age at diagnosis, and cumulative doses of chemotherapeutic agents (methotrexate, ifosfamide). Finally, we investigated the link between functional regional strength, neurocognitive assessments and daily life complaints. In patients relative to controls we observed lower grey matter volumes in cerebellar and frontal areas, as well as frontal cortical thinning. Cerebellar volume and orbitofrontal thickness appeared dose- and age-related, respectively. Cortical thickness of the parahippocampal area appeared lower, only if the group comparison was not adjusted for depression. This region specifically showed lower functional coherence, which was associated with lower processing speed. This study suggests cortical thinning as well as decreased functional coherence in survivors of childhood sarcoma, which could be important for both long-term attentional functioning and emotional distress in daily life. Frontal areas might be specifically vulnerable during adolescence. 
Highlights:

- Frontal, parahippocampal and cerebellar cortical thinning in childhood cancer survivors

- Cortical thinning appear chemotherapy dose-related as well as age-related

61

- Altered functional coherence in patients is associated with lower processing speed

62

63 Keywords: cortical development, intravenous chemotherapy, childhood sarcoma 64 


\section{Introduction}

Evidence for treatment-related changes in psychological functioning of childhood cancer patients is dramatically increasing (Ki Moore et al., 2013; Marusak et al., 2017). However, the underlying neural mechanisms which were suggested remain undetermined (Ahles and Saykin, 2007). Previously, functional neuroimaging was mainly conducted in adult non-CNS cancer patients (Billiet* et al., 2018; Deprez et al., 2012; Hampson et al., 2015; Kesler et al., 2014, 2009). Functional neural changes were not only associated with treatment, but also with adverse life events (Duncan et al., 2015; Yoshikawa et al., 2006), fatigue (Hampson et al., 2015), as well as with anatomical cortical damage (e.g. atrophy) (Hakamata et al., 2007). Given that childhood cancer patients are exposed to both early life stress and cancer treatment during a critical period of neurodevelopment, it is highly important to investigate their neuroanatomical outcomes.

Grey matter (GM) structure in childhood cancer patients has only been investigated in in patients who were treated with cranial radiotherapy (Zhang et al., 2008) and intrathecal (i.e. CNS-directed) chemotherapy (Genschaft et al., 2013; Porto et al., 2008; Tamnes et al., 2015; Zou et al., 2017) (for the treatment of brain tumors and leukemia, respectively). This research showed decreased GM thickness and volumes (Ikonomidou, 2018). Nonetheless, more and more evidence exists for similar anatomical changes in solid tumor patients treated with intravenous (i.e. non-CNS-directed) chemotherapy only (McDonald and Saykin, 2013; Simo et al., 2013). To date, these studies merely included adult cancer populations. Decreased GM volumes and densities were reported in adult patients with breast cancer (Blommaert et al., 2019; Conroy et al., 2013; De Ruiter et al., 2012; Feng et al., 2019; Koppelmans et al., 2012; Lepage et al., 2014; Li et al., 2018; McDonald et al., 2013, 2010), ovarian cancer (Correa et al., 2016), testicular cancer (Amidi et al., 2016) and lung cancer (Pohler, 1992) after chemotherapy. Such differences appeared distributed across the brain (e.g. frontal, parahippocampal, cingulate 
gyrus, and precuneus). In addition, rodent studies also demonstrated neuronal cell death after the administration of chemotherapeutic agents (Wick et al., 2004). Such active degeneration of neurons was also encountered in developing rodent brains (Rzeski et al., 2004). However, cortex-based approaches investigating group differences in cortical thickness and its functional impact have not been studied in childhood cancer patients yet.

Pediatric patients with non-CNS solid tumors often receive high doses of intravenous chemotherapy, which was evidenced to result in white matter alterations (Sleurs et al., 2019, 2018), and altered neurocognitive functioning in patients (Mohrmann et al., 2015) or survivors (Edelmann et al., 2016). These studies demonstrated lower intelligence scores in preschool patients (Willard et al., 2017), as well as long-term complaints of affected memory, processing speed (Edelmann et al., 2016), task efficiency (Kadan-Lottick et al., 2010) and difficulties at school in around a third of non-CNS tumor patients (Mohrmann et al., 2015). However, the amount of cognitive and neuroimaging research in this population remains limited. Besides cognitive changes, mental complaints of discomfort including post-traumatic symptoms (Wilson et al., 2016; Yi et al., 2015), fatigue (Frederick et al., 2016), anxiety and depression (D'Agostino et al., 2016) also highly affect long-term mental health in childhood cancer patients (Friend et al., 2018). In order to investigate cortical abnormalities, we analyzed structural and functional cortical information in adult survivors of childhood sarcomas who had received high doses of intravenous chemotherapy during childhood. Furthermore, we addressed the possible association with doses of chemotherapeutic agents and psychological (self-reported distress-related and neurocognitive) outcomes. Based on previous studies, we hypothesized that anatomical differences could be related to different chemotherapy doses (Seigers et al., 2008; Sugimoto et al., 1995) and to age at diagnosis (Blommaert et al., 2019; Krull et al., 2016; Reddick et al., 2009). It was a priori hypothesized that functional brain differences could be 
related to the behavioral outcomes (Billiet* et al., 2018; Kaiser et al., 2014; Reuter-Lorenz and Cimprich, 2013).

\section{Methods}

\subsection{Participants}

Survivors of childhood bone and soft tissue sarcomas $(n=33)$ who were treated with local treatment (i.e. surgery, radiotherapy) and intravenous chemotherapy only during childhood and adolescence (median 13.32 years old) were recruited in this study (Sleurs et al., 2019). Their treatment consisted of multimodal chemotherapy including high-dose methotrexate either combined with ifosfamide or not (protocols EURAMOS1, RMS2005, NRSTS2005, Euro-Ewing99, MMT95, EORTC2001, IVAD). Patients were at least two years out of treatment. Time since diagnosis ranged between 2.08-19.92 years (mean=9.92, $\mathrm{SD}=4.72$ ). Thirty-four healthy control participants, who were recruited via internal hospital announcements, were age- and gender-matched. More detailed characteristics of the participants were described previously (Sleurs et al., 2019) and provided in Table 1. All participants were aged between 16.14-35.28 years (mean=22.51, SD=3.97) at the moment of acquisition. The study was approved by the Ethical Committee of the University Hospitals Leuven and conducted in accordance with the Declaration of Helsinki.

\subsection{Data acquisition}

First, high-resolution whole brain T1-weighted MRI scans were acquired for morphometrical cortical analyses (i.e. voxel-based (VBM) and surface-based morphometry $(\mathrm{SBM}))$, and for anatomical reference. Acquisition parameters were as follows: MPRAGE, resolution $=.98 \times .98 \times 1.2 \mathrm{~mm}, \mathrm{TR} / \mathrm{TE}=9.6 / 4.6 \mathrm{~ms}, \mathrm{FOV}=192 \times 250 \times 250 \mathrm{~mm}$. Second, resting state functional MR images (rsfMRI) were obtained using a T2*-weighted echo-planar imaging (EPI) sequence. Parameters were: resolution $=3.6 \times 3.6 \times 4 \mathrm{~mm}, \quad \mathrm{TR} / \mathrm{TE}=1700 / 33 \mathrm{~ms}$, $\mathrm{FOV}=230 \times 230 \times 120 \mathrm{~mm} ; 30$ slices with slice thickness $=4 \mathrm{~mm}$. Both scan sequences were 
obtained within one scanning session on a 3T Philips Achieva MRI scanner with a 32-channel phased-array head coil.

Questionnaires were obtained assessing self-reported emotional distress (anxiety

142 (STAI) and depression (BDI)), executive functioning (BRIEF-A), cognitive failure (CFQ),

143 quality of life (PedSql) and the Hollingshead Four Factor Index of Socio-Economic Status

144 (SES). This SES score is based on the participant's parent's education (rated on a 7-point scale)

145 and occupation (rated on a 9-point scale). An extensive neurocognitive test battery covered the

146 domains of intelligence (WAIS), Auditory Verbal Learning Test (AVLT) and Rey Visual

147 Design Learning Test (RVDLT), as well as computerized attention and executive tasks

148 (Amsterdam Neuropsychological Task battery, ANT). Based on previous behavioral group

149 comparisons in this cohort (Sleurs et al., 2019), only significant measures were extracted and

150 analyzed in the current study. These measures included processing speed (based on the 'coding'

151 and 'symbol search' subtests of the WAIS-IV), working memory (based on the 'digit span' and

152 'arithmetic' subtests of the WAIS-IV), and attentional reaction times (ANT computerized tasks)

\subsection{Data preprocessing and statistics}

T1-weighted MRI images were preprocessed (see Supplementary Materials) and processed in CAT12 (SPM12) toolbox for VBM and SBM investigations using standard settings (Gaser and Dahnke, 2016). Resting state fMRI images were preprocessed (see details in Supplementary Materials) and subject-specific functional connectomes were constructed consisting of 84 nodes (see Figure 1 and Supplementary Materials) based on positive partial correlations (in-house matlab scripts). First, whole-brain anatomical group comparisons (patients vs. controls) were performed for grey matter volume (GMV) at voxel-level (VBMbased) using a GLM model (patients $<$ controls and vice versa; with SES and total intracranial volume as covariates). Second, cortical thickness (SBM-based) was compared between both 
it is known that depression can have an impact on cortical density and thickness (Jaworska et al., 2016; Schmaal et al., 2017; Thompson et al., 2014). In order to account for the effect of depression scores (which were significantly different between the 2 groups, see Supplementary Materials) (Sleurs et al., 2019), these models were expanded including the predictor of depression (BDI) as covariate. Group comparisons were assumed significant if both FWEcorrected p-values at cluster-level reached $<.05$, and uncorrected p-values at voxel-level reached $<.001$.

Fourth, functional efficiency values were calculated at whole-brain level (i.e. global efficiency), local level (i.e. local efficiency) and nodal level (i.e. nodal strength) using the Brain Connectivity Toolbox (BCT) (Rubinov and Sporns, 2010). Nodal values were assessed for a priori selected regions only (i.e. regions with significant differences in GMV or cortical thickness). All abovementioned values were compared between patients and controls for a range of network density thresholds (0-50\%) (Achard and Bullmore, 2007).

Finally, structural measures of GMV and cortical thickness of significant regions were correlated with chemotherapy doses of methotrexate and ifosfamide (corrected for age at diagnosis), as well as with age at diagnosis (corrected for the cumulative doses of the two agents), to address their association with the extent of structural damage. Functional nodal strength of significant nodes was correlated with the abovementioned behavioral measures, in order to address the association between functional activity and behavioral outcome (i.e. BDI,

STAI, CFQ, working memory, processing speed). These partial correlations were calculated within the survivor group, and assumed significant at $\mathrm{p}<.05$.

3. Results 
for depression, these regions remained significant and a third region appeared in the cerebellum

190 (see Figure 2, panel B). No significant differences were found in the opposite direction (i.e. 191 patients $>$ controls).

192 Comparably, based on SBM, cortical thickness appeared lower in patients than in controls in 193 the right orbitofrontal region ( 2 clusters), left medial frontal region and left parahippocampal 194 region (see Figure 3, panel A). After additional correction for depression, all frontal regions 195 remained significant. By contrast, the parahippocampal region was no longer significant, while a second cluster in the left medial frontal appeared significant after adding this predictor (see Figure 3, panel B). Again, no regions were detected with higher cortical thickness in patients 198 than controls.

\subsection{Functional coherence: nodal strength}

Based on the functional connectomes, global and local efficiency measures across the entire brain were not significantly different between patients and controls. However, at a regional level, functional nodal strength (calculated for the regions showing significant differences in VBM and SBM) appeared significantly decreased in the left parahippocampal volume in patients compared to controls, which remained the case for any network threshold (Figure 4A) and after normalisation (Figure 4B. Furthermore, nodal degree (i.e. the number of functional connections) of this region was also significantly decreased when thresholding for the strongest connections ( $<10 \%$ network density, Figure 4C). Group average partial correlations between this region and other brain regions are shown in Figure 4D; which were thresholded in 4E.

Cerebellum, left and right rostral middle frontal region and left lateral orbitofrontal region did not show any significant differences in functional nodal strength between patients and controls. 


\subsection{Risk and outcome of cortical thickness and functional nodal strength}

Partial correlations between cortical measures (GMV and cortical thickness) and

215 cumulative chemotherapeutic (methotrexate and ifosfamide) doses, corrected for age at

216 diagnosis (and vice versa) were calculated ( $\mathrm{n}=33$; Table 2). These yielded significant positive

217 correlations between the cortical thickness of the right lateral orbitofrontal and age at diagnosis

218 (Table 2). This indicates that younger age at diagnosis is associated with smaller cortical

219 thickness of the right lateral orbitofrontal region. Furthermore, chemotherapy dose appeared to

220 be associated with the cerebellar grey matter volume. Finally, correlations between behavioral

221 outcomes $(n=33)$ and parahippocampal nodal strength were significant for processing speed

222 (see Table 3).

223 


\section{Discussion}

To our knowledge, this is the first study integrating structural and functional neuroimaging of the grey matter in pediatric non-CNS cancer survivors. We demonstrated lower grey matter volume and cortical thickness in frontal areas, and lower grey matter volume in the cerebellum, which remained after correction for depression. The latter was related to the dose of chemotherapy received, while cortical thickness of the right orbitofrontal area was related to age at cancer diagnosis. The additional predictor of depression seemed to play a role in differences in cortical thickness of the parahippocampal area. Only for this region, a significantly lower nodal strength was observed in patients, which was associated with lower processing speed in patients.

\subsection{Structural cortical differences and risk factors}

The medial- and orbitofrontal brain regions showed both lower GM volume as well as lower cortical thickness. This finding suggests both cortical thinning of the cortical layer, as well as decreased GM density in childhood cancer survivors. As frontal brain areas develop later during childhood, this might explain the significant age-related effect in the orbitofrontal area. Previously, Hakamata and colleagues (2007) also showed lower GM volumes in the orbitofrontal regions, but specifically in adult cancer survivors suffering from post-traumatic stress disorders (Hakamata et al., 2007).

By contrast, the parahippocampal area only showed lower cortical thickness, when not corrected for depression scores. Consequently, depression rates might partially explain lower cortical parahippocampal cortical thickness more in general (correlations see Supplementary Materials). As BDI values were higher in patients (Sleurs et al., 2019), lower cortical thickness in this group can partially be explained by their higher depression scores. As a consequence, long-term depressive symptoms can be an additional risk factor. In cancer patients specifically, one study by Yoshikawa and colleagues (2007) demonstrated smaller amygdala volumes in 
breast cancer patients who had experienced a depressive episode (Yoshikawa et al., 2006).

250 Earlier studies also demonstrated age-related cortical volumes and decreased thickness of

251 orbitofrontal cortex and temporal areas in non-cancer patients who suffer from major depressive

252 disorders (Jaworska et al., 2016; Schmaal et al., 2017). These researchers hypothesized age-

253 dependent stress-related remodeling. In childhood cancer patients in specific, traumatic

254 experiences could yield similar developmental changes. Recently, such neurobiological 'double

255 hit' hypothesis (i.e. effect of treatment combined with stress) was posed by Marusak and

256 colleagues (2017), suggesting specific vulnerability of the hippocampus (Marusak et al., 2017).

257 Our neuroimaging findings seem to be in line with this hypothesis. Hence, implementation of

258 affect-related measures (both subjective and biochemical markers) in future neuroimaging

259 research, could yield additional insights. BDI was selected as covariate in this study, since it is

260 known to be associated with cortical thickness (Schmaal et al., 2017) and it was significantly

261 different between patients and controls (see Supplementary Materials). Furthermore, it should

262 be noted that only 5 patients versus 2 controls reached the mild to moderate depression level

263 (i.e. total BDI score between 10-18) (Beck et al., 1988). Severe depression levels were never

264 reached. Average group scores did not reach the clinical cutoff $(M$ controls $=3.68, M$ patients $=6.16)$.

265 So, increased risk according to depressive symptoms could be linear, but in this cohort they remained within the normal range.

With regard to age-dependency, we specifically showed younger age at diagnosis to be associated with lower frontal cortical thickness. Frontal brain areas are known to develop later throughout neurodevelopment (Giedd et al., 1999; White et al., 2010). This is thought to be an adaptive process of selective pruning (Kharitonova et al., 2013). Given that the majority of our

271 patients were treated during adolescence, this finding suggests that these frontal regions might

272 be specifically vulnerable to induced neurotoxicity and consequent delay in development. 
Besides structural differences in the cortex, we previously evidenced white matter

alterations in the same cohort. Visually observable lesions were detectable in $9 / 33$ patients.

Unfortunately, this sample is too small to perform subset analyses (Sleurs et al., 2019). Furthermore, even in non-lesioned patients, more subtle white matter changes could occur, as evidenced in a diffusion-weighted MRI analysis (Sleurs et al., 2018).

\subsection{Functional cortical differences and functional outcomes}

In addition to smaller cortical thickness of the parahippocampal region in survivors compared to controls, this region also showed lower functional nodal strength. Furthermore, this appeared to correlate with processing speed values in the survivor subgroup. Given that the parahippocampal cortical thickness appeared lower in patients, only as long as it was not controlled for depression, this region could potentially play a role in depressive scores (or be affected by it) as well, which also is associated with lower processing speed (Lähteenmäki et al., 1999). Similarly, an EEG study in childhood cancer survivors has demonstrated lower processing speed in an auditory attention task (Lähteenmäki et al., 1999), which is consistent with our behavioral findings. However, this study only included 19 survivors, treated for leukemia with cranial radiotherapy. Furthermore, EEG has lower spatial resolution compared to MRI. Our study extends these previous findings with decreased rsfMRI connectivity (of the parahippocampal area) in survivors of solid tumors who did not receive any cranial radiotherapy.

The fact that cortical thickness as well as nodal strength of the left parahippocampal area were lower in patients, might suggest a structural as well as functional sensitivity of this region. We note that only five regions were extracted for the functional nodal comparisons, based on the voxel- and surface-based results, to limit the number of statistical tests. However, one could hypothesize that functional strength is affected more globally, and the parahippocampal strength difference is not as specific as was hypothesized. Nonetheless, post- 
hoc, non-corrected group comparisons of all regions demonstrated the parahippocampal region to be one of the limited number of regions showing lower functional strength (see Supplementary Materials). This strengthens the hypothesis of a specific vulnerability of the

301 parahippocampal area to treatment-, but also negative affect-related neurotoxicity. Previously, 302 studies have shown depression-related atrophy of the hippocampus (Sapolsky, 2001). The functional nodal strength of the parahippocampal area was lower in survivors and moreover associated with survivors' processing speed. Although this region is mainly associated with memory encoding and retrieval, hippocampal and parahippocampal subregions are proposed to relate to multiple functional systems and behavioral outcomes (Aggleton, 2012; Rothschild et al., 2017). Given that the parahippocampal strength was related to processing speed, the parahippocampal-prefrontal system could be of particular interest. Thresholded connectivity of the left parahippocampal area showing a stronger connection with the right orbitofrontal area in controls, is in line with this hypothesis.

312 information processing speed (Rock et al., 2014). Given that the survivor group showed 313 elevated depression scores (see Supplementary Materials), this could specifically affect processing speed. In this regard, future imaging techniques with higher resolution could be promising to image (sub)cortical brain subregions in more detail. 


\subsection{Absence of network efficiency differences}

Functional efficiency measures were not significantly different between patients and controls. Similar to our findings, Kesler and colleagues (2017) recently demonstrated no group differences in global efficiency in breast cancer patients, and only subtle differences in local efficiency (Kesler et al., 2017). Based on structural networks, Amidi and colleagues (2017) also did not encounter global efficiency differences, but did encounter lower local efficiency in testicular cancer patients (Amidi et al., 2017). This suggests that (functional) networks are probably not profoundly reorganized, but overall functional coherence (e.g. of the parahippocampal area) might be lower in patients than controls. The fact that we encountered lower coherence of the parahippocampal area at group level, could demonstrate a particular sensitivity of this region. Furthermore, its association with processing speed becomes particularly interesting, given that this cognitive function is related to multiple other psychological symptoms as well (e.g. depression, fatigue, ...).

\subsection{Limitations and future directions}

We only report lower GMV in cerebellar regions, but cannot provide information about the thickness of the cerebellar cortical layer. Unfortunately, available techniques are unable to derive cortical thickness of the cerebellum at the current resolution. Higher resolution and advanced processing techniques might yield the possibility for surface-based investigations in cerebellar subareas as well. Given that the groups significantly differed in BDI and SES scores, it is inherently challenging to disentangle the group difference that can partly be due to cancer therapy and partly due to elevated depression or lower SES scores. With regard to BDI, both models including and excluding BDI as covariate were therefore reported. Future studies could also include a group of clinically depressed cancer patients as a control group, or enlarge the group to estimate the additional effects of depression in more detail. With regard to SES scores, 
values (i.e. we checked the main effect of SES in the original model on GM volume and cortical thickness; and analyzed the data after exclusion of SES outliers) (see Supplementary Materials). These analyses confirmed that no SES main effects were associated with cortical volumes or thickness, and that group effects remained significant if SES was matched more closely (based on smaller subgroup comparisons). Based on these results, we conclude robust effects of the cancer or its treatment, even after controlling for SES effects. Future studies ideally match SES by recruiting a control for each patient at individual level.

Furthermore, to estimate functional coherence in the brain, we calculated positive partial correlations between atlas-based areas. The calculation of partial correlations is currently a standard implemented approach to estimate coherence between regions, as they would remove sources of shared variance in oscillations with other brain areas (Murphy and Fox, 2017). Furthermore, we regressed out the cerebrospinal fluid and white matter signals and denoised the data from components classified as noise. However, we did not regress out global BOLD signal, and ignored anti-correlations (i.e. negative correlations). For both decisions pros and cons are currently under debate (Murphy and Fox, 2017). Regression of the global signal might help remove non-neural related covariance between regions (e.g. movement, respiration). However, it can also result in spurious artefactual anti-correlations. In this study, we focused on positive correlation values in the connectomes only, since it remains unclear whether anticorrelations in BOLD signal are biologically meaningful or not. In addition, to investigate excitatory and inhibitory effects of regions to other brain regions, dynamic analyses of the BOLD signals could provide more detailed temporal information, as compared to partial correlations. Partial correlations provide a static measure of coherence of regions across the entire fMRI acquisition ( $7 \mathrm{mins}$ ). However, dynamic properties of these networks (rather than the size or strength of nodes and networks) such as efficient switching, might lead to a more complete understanding of treatment-induced neurocognitive impact. 
We specifically focused on nodal strength measures of brain regions showing lower

368 GMV or cortical thickness. Hence, we did not investigate connectivity between specific regions. Although we thus could not clarify localized "connection" differences, an investigation of all the included partial correlations between regions to regions, would have resulted in 6972 connection comparisons (based on 84 nodes). This would have elevated the risk for type I errors. Therefore, we focused on nodal strength of each node as a more robust measure, instead of specific ROI-to-ROI correlations. Nevertheless, we cannot exclude the possibility of brain area-specific differences in functional coherence, which did not necessarily differ in nodal strength. Using our method, specific differences in connectivity could therefore have been missed. Furthermore, we cannot extract the possible interdependency between the anatomical and functional data, which might have affected the results of the rsfmri analyses, since the ROIs were based on the anatomical group comparison results.

Finally, this study investigated a group of solid tumor survivors, who were treated according to different chemotherapeutic protocols, with different time intervals since treatment. This heterogeneity in timing could have affected the results, since both degenerative and regenerative processes might be involved. Such developmental changes cannot be deduced from this study due to the cross-sectional design. As these treatments, different ages at diagnosis and at participation result in a heterogenous population, investigations of more complicated interaction effects (e.g. between chemotherapeutic agents, neurodevelopmental effects and other individual risk factors) require larger sample and longitudinal studies.

\section{Conclusion}

This is the first neuroimaging study of childhood cancer survivors integrating structural and functional grey matter investigations. This study suggested long-term cortical alterations of cerebellar, frontal and the parahippocampal area. With regard to the parahippocampal region, both cortical thickness and functional strength appeared lower in patients, of which the latter 
was associated with lower processing speed. Furthermore, patients diagnosed at younger age showed more cortical thinning in orbitofrontal areas. Based on this cohort study, we assume that treatment- or negative affect-related neurotoxicity to the grey matter could be agedependent, with frontal and limbic areas being vulnerable during adolescence. In addition to previous adult oncology studies reporting therapy-related neurotoxicity, our findings of structural brain alterations, complemented with functional differences in the parahippocampal region, supports the hypothesis of chemotherapy dose-, affect- and age-related remodeling of neurodevelopment in childhood cancer.

\section{Acknowledgements}

We are grateful to Ahmed Radwan for sharing his neuroradiological knowledge. In addition, we thank Floris Pelkmans and Iris Elens for their help during data acquisition and sharing data. This work was supported by the Kinderkankerfonds Leuven. In addition, JB acknowledges support from Research Foundation Flanders (FWO, grant no. 11B9919N). DB acknowledges support from the Wellcome/EPSRC Centre for Medical Engineering [WT 203148/Z/16/Z]; the National Institute for Health Research (NIHR) Mental Health Biomedical Research Centre (BRC) at South London and Maudsley NHS Foundation Trust and King's

College London; and the NIHR-BRC at Guys and St Thomas' NHS Foundation Trust and King's College London.

\section{Compliance with ethical standards}


418
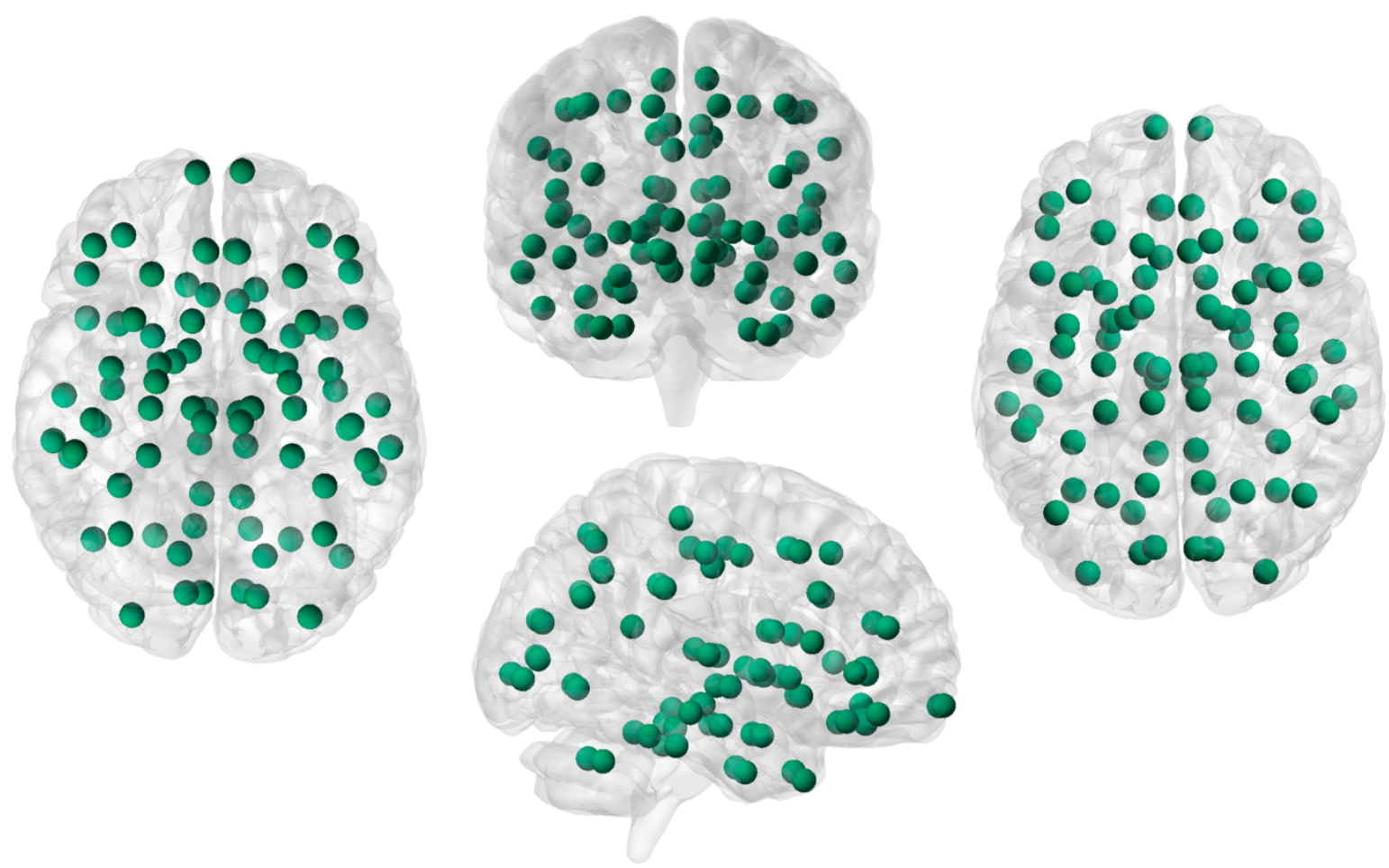

420 Figure 1. Included nodes for connectome construction based on the Desikan-Killiany atlas

421 Note. 66 nodes of the Desikan-Killiany atlas were complemented with 9 additional nodes from

422 the whole-brain freesurfer parcellation in each hemisphere, resulting in 84 nodes in total. 


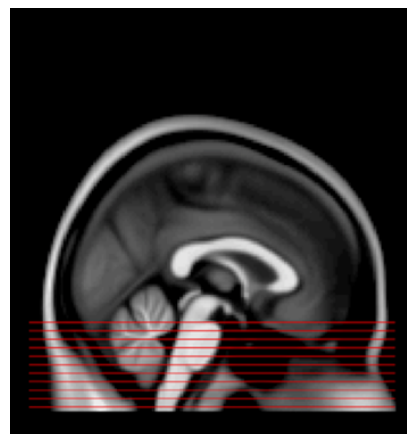

\section{A}

P-values
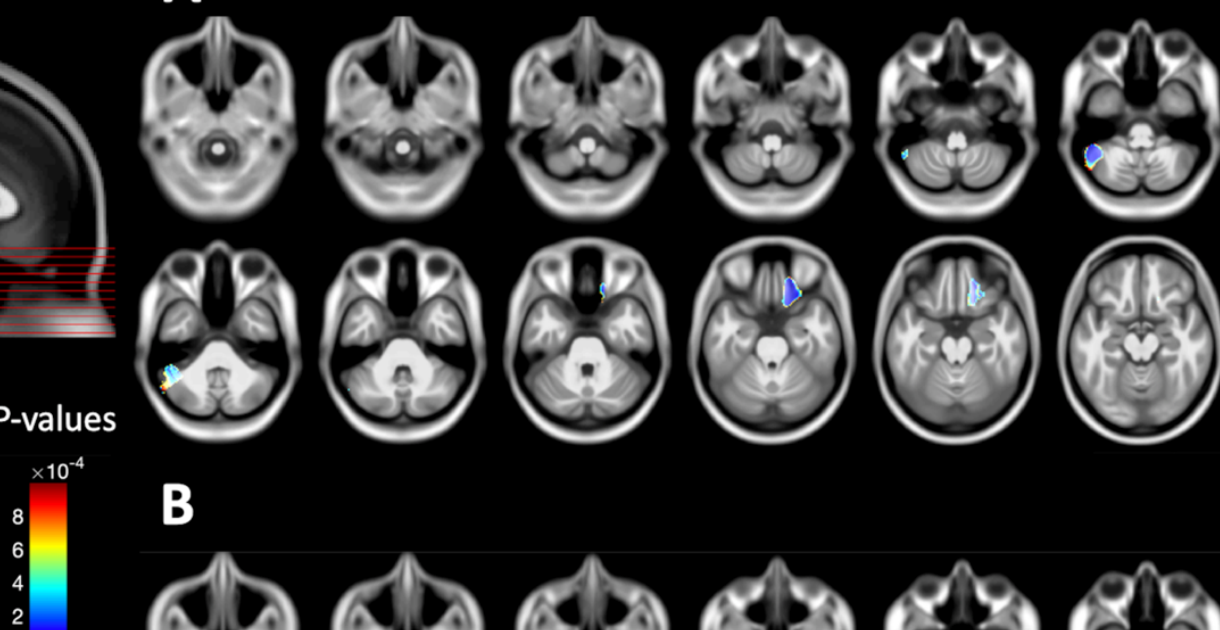

B
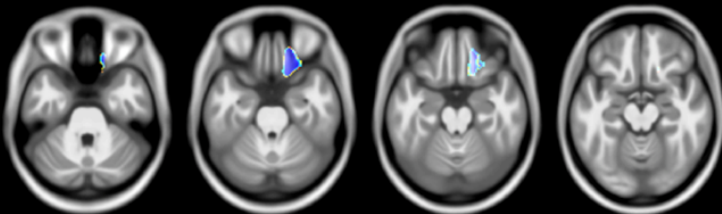

$\mathbf{R}$
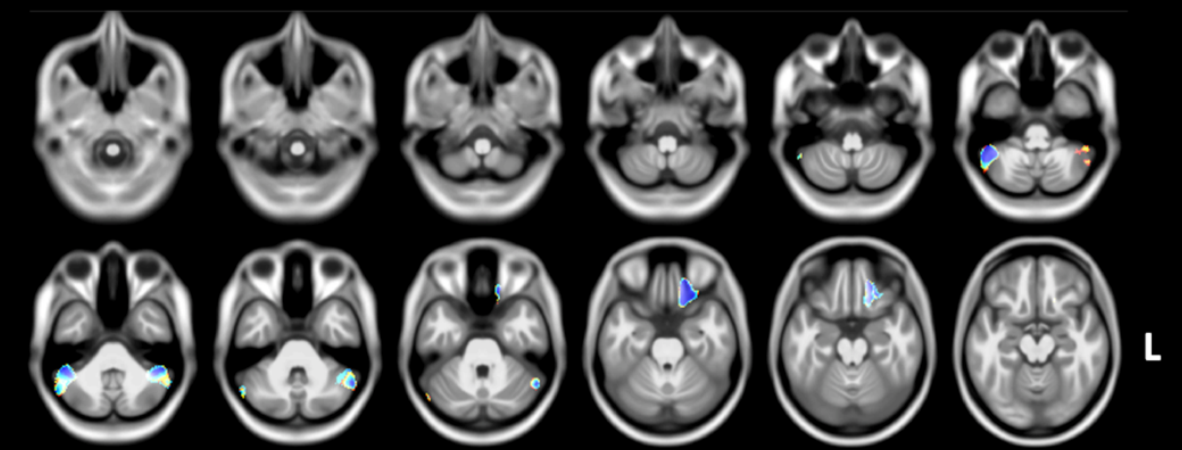

424 Figure 2. ANOVA group comparison results of GMV (voxelwise p-values) patients < controls

425 Note. Panel A depicts the significant group difference patients $<$ controls ( $p$-values), corrected 426 for SES and total intracranial volume; panel B shows the results after additional correction for

427 BDI. Regions were assumed significant if both FWE-corrected $\mathrm{p}$-values reached $<.05$ at cluster428 level, and uncorrected p-values reached $<.001$ at voxel-level. 

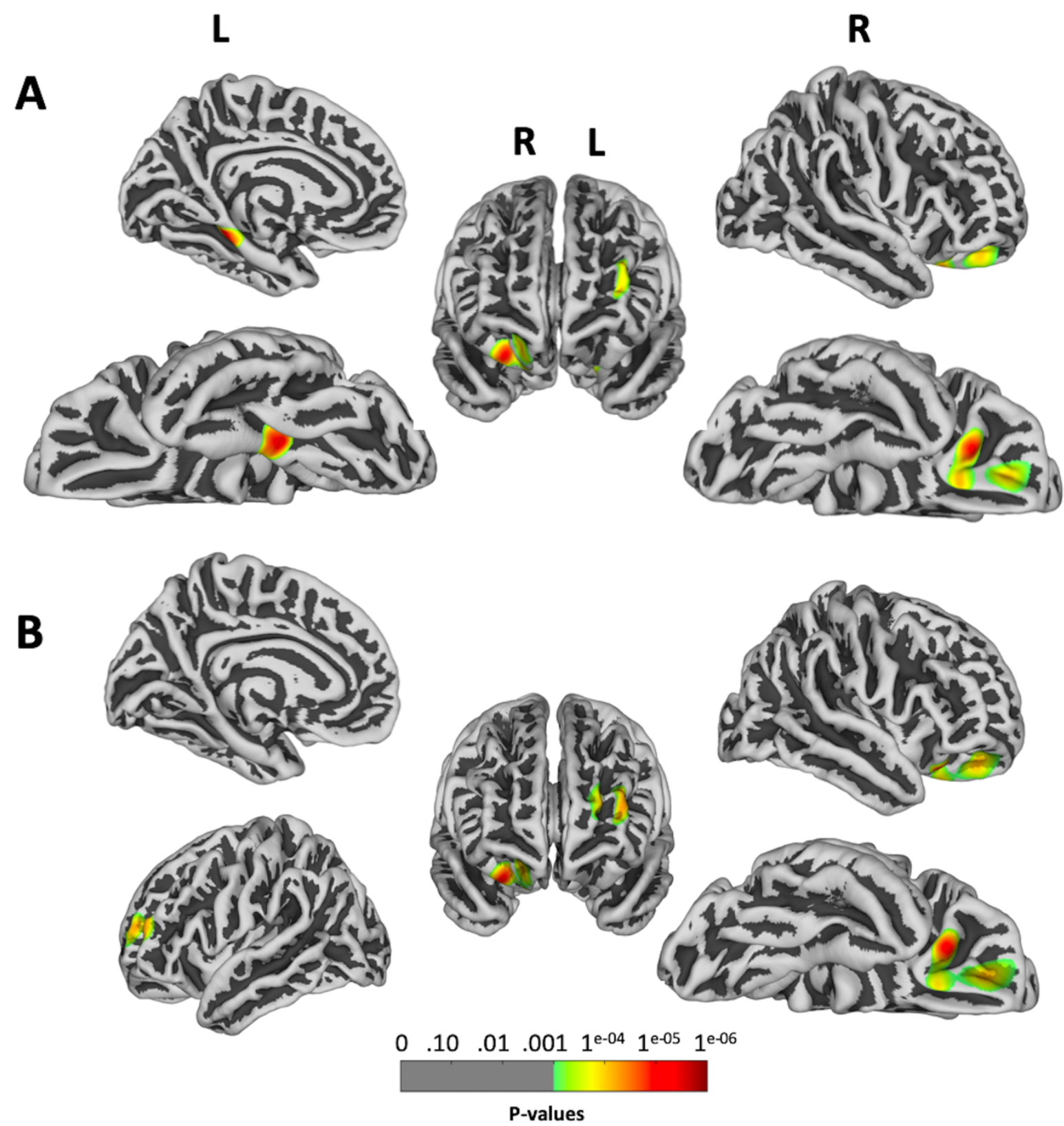

431

P-values

Figure 3. ANOVA group comparison results of cortical thickness ( $p$-values) patients $<$ controls

433 Note. Panel A depicts the significant group difference patients < controls, corrected for SES 434 and age at assessment; panel B shows the results after additional correction for BDI. Regions 435 were assumed significant if both FWE-corrected p-values reached $<.05$ at cluster-level, and 436 uncorrected p-values reached $<.001$ at voxel-level. 
A

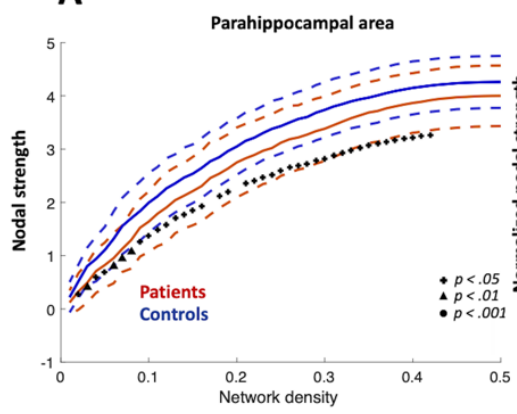

B
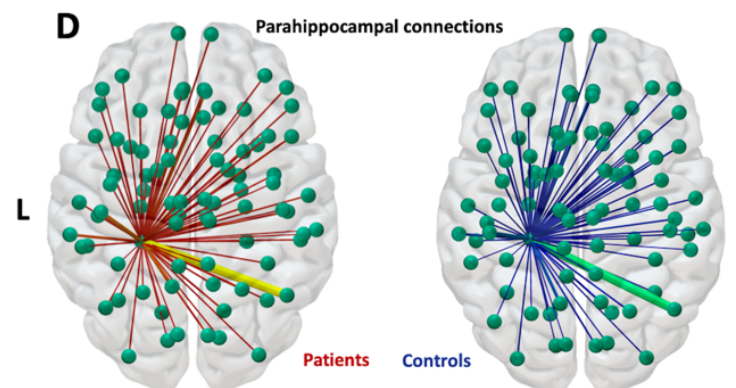
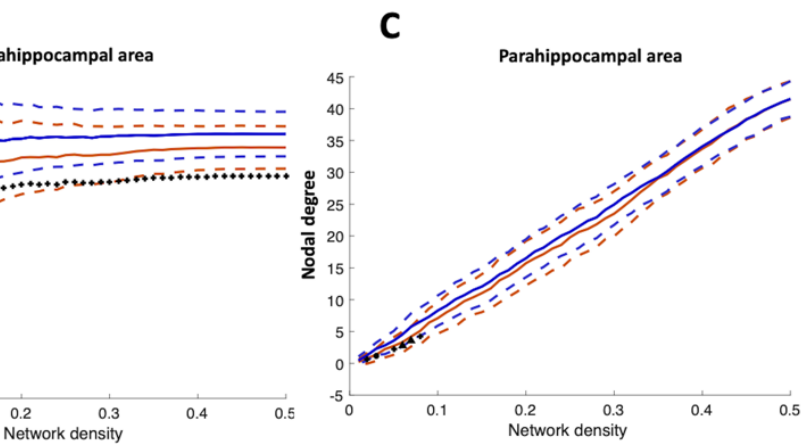

E

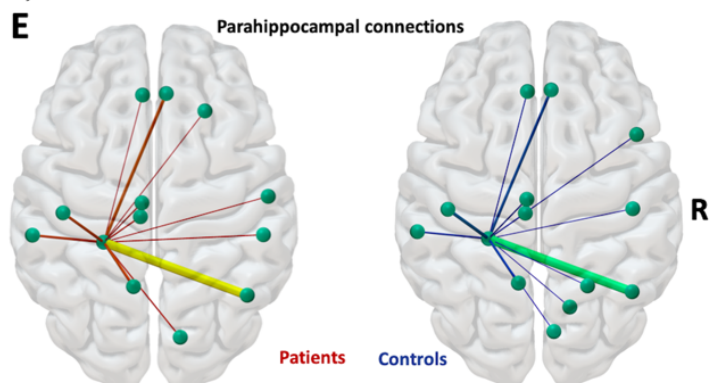

437

438

439

440

441

442

443

444

445

446

447

448

449

450

451

452

453

454

455

456

457

458

Figure 4. ANOVA group analysis results of functional strength values in the left parahippocampal region

Note. Panel A depicts the results of the ANOVA group comparison of nodal strength values between patients and controls, for a range of network densities between 1-50\% thresholds. This network density range represents the proportionally thresholded network with an increase of $.1 \%$ (e.g. network density .1 means the subject-specific networks were thresholded at $10 \%$ strongest connections). Continuous lines represent group-specific average values of nodal strength, while dotted lines represent group-specific values of the mean $+/-1$ standard deviation. Panel B depicts the results of the ANOVA group comparison of normalized nodal strength values. These values represent the nodal strength of the parahippocampal region, proportional to the entire subject-specific network. Panel $\mathrm{C}$ depicts the results of the ANOVA group comparison of nodal degree values (i.e. the total number of existing connections). Panel D represents all group average partial correlations of the parahippocampal region, patients are depicted in red, control participants in blue. Panel E represents the thresholded (i.e. correlations $>$.05) group average partial correlations of the parahippocampal region. This figure shows correlations that remain in both subgroups, and some that remain in only one of them. More specifically, correlations between the left parahippocampal region and right orbitofrontal region, right pericalcarine region, right superior temporal area, and right supramarginal region remained at this threshold in the control participant group, but not in the patient subgroup; whereas correlations that remain in patients but not in controls include the left parahippocampal 
459 region with right cuneus, lingual region, pars opercularis, superior parietal and transverse 460 temporal region.

461

462 
Table 1. Participant characteristics

\begin{tabular}{|c|c|c|c|c|c|c|}
\hline DIAGNOSIS & \multicolumn{2}{|c|}{ OSTEOSARCOMA } & EWING & \multicolumn{2}{|c|}{$\begin{array}{c}\text { RHABDOMYOSARC } \\
\text { OMA }\end{array}$} & $\begin{array}{c}\text { NON- } \\
\text { RHABDOMYOSARCO } \\
\text { MA SOFT TISSUE } \\
\text { SARCOMA }\end{array}$ \\
\hline $\begin{array}{l}\text { TREATMENT } \\
\text { NUMBER } \\
\text { AGE DIAGNOSIS }\end{array}$ & $\begin{array}{l}\text { EORTC2001 } \\
3 \\
12-15 y\end{array}$ & $\begin{array}{l}\text { EURAMOS1 } \\
13 \\
10-18 \mathrm{y}\end{array}$ & $\begin{array}{l}\text { EuroEW99 } \\
7 \\
12-15 y\end{array}$ & $\begin{array}{l}\text { MMT95 } \\
5 \\
5-15 y\end{array}$ & $\begin{array}{l}\text { RMS2005 } \\
3 \\
9-16 y\end{array}$ & $\begin{array}{l}\text { NRSTS2005 } \\
2 \\
11-14 y\end{array}$ \\
\hline $\begin{array}{l}\text { TIME SINCE } \\
\text { THERAPY } \\
\text { MTX }\left(\mathbf{G} / \mathbf{M}^{2}\right)\end{array}$ & $\begin{array}{l}14-15 y \\
45(n=1)\end{array}$ & $\begin{array}{l}2-13 y \\
96-144 \\
(n=13)\end{array}$ & $4-15 y$ & $12-20 y$ & $4-8 \mathrm{y}$ & $2-20 y$ \\
\hline $\begin{array}{l}\text { IFOSFAMIDE/ } \\
\text { CYCLOPHOSPH } \\
\text { AMIDE }\left(\mathbf{G} / \mathbf{M}^{2}\right) \\
\text { DOXORUBICIN } \\
\left(\mathrm{MG} / \mathbf{M}^{2}\right)\end{array}$ & $36(n=1)$ & $60-87(\mathrm{n}=4)$ & $\begin{array}{l}102(\mathrm{n}=6) / \\
12(\mathrm{n}=2) \\
180-360\end{array}$ & $\begin{array}{l}36-54 \\
(n=4)\end{array}$ & $\begin{array}{l}24-102 \\
375(n=1)\end{array}$ & $\begin{array}{l}33-36 \\
210-240\end{array}$ \\
\hline \multirow[t]{2}{*}{$\begin{array}{l}\text { CISPLATIN } \\
\left.\text { (MG/M }^{2}\right) \\
\text { CARDIOTOXICIT } \\
\text { Y } \\
\text { NEUROTOXICIT } \\
\text { Y } \\
\text { WM FAZEKAS } \\
\text { RATING }\end{array}$} & $\begin{array}{l}600 \\
1\end{array}$ & $240-480$ & 0,1 & $\begin{array}{l}1 \\
0,1\end{array}$ & $\begin{array}{l}1 \\
0,1\end{array}$ & \\
\hline & \multicolumn{3}{|c|}{ PATIENTS } & \multicolumn{3}{|c|}{ CONTROLS } \\
\hline $\begin{array}{l}\text { AGE AT } \\
\text { ASSESSMENT } \\
\text { SES }\end{array}$ & \multicolumn{3}{|c|}{$23.04(4.31)$} & \multicolumn{3}{|c|}{$22.12(3.60)$} \\
\hline BRIEF & \multicolumn{3}{|c|}{$54.60(9.57)$} & \multicolumn{3}{|c|}{$52.26(9.34)$} \\
\hline PEDSQL & \multicolumn{3}{|c|}{1645 (280.19) } & \multicolumn{3}{|c|}{$1913.71(226.37)$} \\
\hline STAI & \multicolumn{3}{|c|}{$38.83(12.15)$} & \multicolumn{3}{|c|}{$34.39(8.13)$} \\
\hline BDI & \multicolumn{3}{|c|}{$6.16(4.95)$} & \multicolumn{3}{|c|}{$3.68(3.49)$} \\
\hline CFQ & \multicolumn{3}{|c|}{$36.97(16.08)$} & \multicolumn{3}{|c|}{$32.35(50.57)$} \\
\hline FSIQ & \multicolumn{3}{|c|}{$100.58(13.86)$} & \multicolumn{3}{|c|}{$109.91(11.76)$} \\
\hline PRI & \multicolumn{3}{|c|}{$102.24(16.01)$} & \multicolumn{3}{|c|}{$101.74(13.13)$} \\
\hline VCI & \multicolumn{3}{|c|}{$100.55(13.63)$} & \multicolumn{3}{|c|}{$108.97(13.63)$} \\
\hline WM & \multicolumn{3}{|c|}{$97.94(13.58)$} & \multicolumn{3}{|c|}{$109.12(13.92)$} \\
\hline PS & \multicolumn{3}{|c|}{$99.45(14.02)$} & \multicolumn{3}{|c|}{$107.38(14.23)$} \\
\hline AVLT & \multicolumn{3}{|c|}{$62.63(7.07)$} & \multicolumn{3}{|c|}{$62.58(5.93)$} \\
\hline RVDLT & \multicolumn{3}{|c|}{$54.67(10.19)$} & \multicolumn{3}{|c|}{$58.52(7.15)$} \\
\hline ANT BASELINE & \multicolumn{3}{|c|}{$-.49(.79)$} & & & $(.65)$ \\
\hline
\end{tabular}

Note. The upper panel presents demographic and treatment data of participating patients. The lower panel represents mean and standard deviation values of age, SES and cognitive values for patients vs. controls. ANT=Amsterdamse Neuropsychologische Taken (baseline T-score), AVLT=Auditory Verbal Learning Test (learning phase), BDI=Beck Depression Inventory, BRIEF=Behavioral Rating Inventory of Executive Function (T-scores), CFQ = Cognitive Failure Questionnaire (raw scores, max=5(rating)*25(items):125), PEDSQL $=$ Pediatric Quality of Life inventory (reverse scores (Varni 2017)), MTX=methotrexate, WM=white matter, FSIQ=Full Scale IQ (standardized, M=100, SD=15), PRI=Perceptual Reasoning Index (standardized, $\mathrm{M}=100, \mathrm{SD}=15$ ), $\mathrm{VCI}=$ Verbal Comprehension Index (standardized, $\mathrm{M}=100, \mathrm{SD}=15$ ), WM=Working Memory (standardized, $\mathrm{M}=100, \mathrm{SD}=15$ ), SES=Socio-economic Status (the Hollingshead Four Factor Index of Social Status), $\mathrm{PS}=$ Processing Speed (standardized, $\mathrm{M}=100, \mathrm{SD}=15$ ), $\mathrm{STAI}=$ State-Trait Anxiety Inventory (raw scores, $\max =4$ (rating)*20(items):80), RVDLT=Rey Visual Design Learning Test (learning phase). 
478 Table 2. Partial correlations between cortical volume and cortical thickness (of VBM- and

479 SBM-selected regions, resp.) with chemotherapy dose (corrected for age at diagnosis) and age

480 at diagnosis (corrected for chemotherapy dose) ( $n=33$ patients)

\begin{tabular}{lllll}
\hline & \multirow{2}{*}{ Region of interest } & \multicolumn{3}{c}{ Partial correlation (p-value) } \\
\cline { 3 - 5 } & & cumulative & cumulative & age at \\
& & MTX dose & IFO dose & diagnosis \\
\hline Grey matter & Cerebellar cluster & $-.372(.039) *$ & $.454(.010) *$ & $-.291(.119)$ \\
volume & Frontal cluster & $-.124(.506)$ & $-.016(.934)$ & $-.034(.857)$ \\
$($ VBM $)$ & & & & \\
\hline Cortical & Left rostral middle frontal & $.071(.704)$ & $.049(.795)$ & $-.111(.559)$ \\
thickness & Right rostral middle frontal & $.147(.431)$ & $.001(.996)$ & $-.015(.936)$ \\
$(\mathrm{SBM})$ & Right lateral orbitofrontal & $.149(.422)$ & $-.068(.718)$ & $.388(.034) *$ \\
\hline
\end{tabular}

481

482

483

484

485

486

487

488

489

490
Table 3. Bivariate correlations between functional nodal strength of the parahippocampal region and behavioral outcome measures in patients $(n=33)$

\begin{tabular}{ll}
\hline Scale & $\begin{array}{l}\text { Correlation (p-value) } \\
\text { with parahippocampal nodal strength }\end{array}$ \\
\hline PedSql & $-.011(.957)$ \\
STAI & $.163(.399)$ \\
BDI & $.262(.170)$ \\
CFQ & $.059(.761)$ \\
WAIS working memory & $-.080(.675)$ \\
WAIS processing speed & $.438(.015)^{*}$ \\
ANT reaction time baseline & $-.002(.991)$ \\
ANT reaction time divided attention & $-.334(.076)$ \\
ANT set shifting: inhibition effect & $-.326(.085)$ \\
ANT stability reaction time sustained attention & $-.055(.782)$ \\
\hline
\end{tabular}

Regions of interest were selected based on the VBM and SBM results (significant regions with different grey matter volume and cortical thickness at group level, respectively).

Note. ${ }^{*}$ indicates $\mathrm{p}<.05 . \mathrm{BDI}=$ Beck Depression Inventory, $\mathrm{CFQ}=$ Cognitive Failure

Questionnaire, STAI = State-Trait Anxiety Inventory, WAIS $=$ Wechsler Adult Intelligence Scale, ANT $=$ Amsterdam Neuropsychological Tasks. 


\section{Supplementary Materials:}

492

493

494

495

496

497

498

499

500

501

502

503

504

505

506

507

508

509

510

511

512

513

514

\subsection{Data preprocessing}

First, T1-weighted MRI images were skull-stripped using FSL5 brain extraction (Jenkinson et al., 2002), and bias field corrected using ANTs (Tustison et al., 2010). These images were further processed in CAT12 (SPM12) toolbox for VBM and SBM investigations using standard settings (Gaser and Dahnke, 2016). In this process, images were non-linearly registered to MNI template space and segmented using a 6-tissue probability map (including the grey and white matter, and CSF). For VBM, these were smoothed with a kernel of $8 \mathrm{~mm}$, and modulated (or multiplied) by the Jacobian determinants. For SBM, surface data were extracted from the segmented images, mapped to the template cortical surface, and smoothed with a kernel of 15 $\mathrm{mm}$. The grey matter maps were further processed for grey matter volume (based on VBM) and cortical thickness estimations (based on SBM).

Second, resting state fMRI images were preprocessed using FSL (realignment, brain extraction, spatial smoothing of $2 \mathrm{~mm}$, intensity normalization). Signal denoising and nuisance regression of the CSF and white matter were performed, using FSL and its add-on ICAAROMA toolbox (Pruim et al., 2015). Finally, a high pass filter of .01Hz was applied. Using Freesurfer, subject-specific parcellations were estimated based on the T1-weighted MRI image, applying the Desikan-Killiany atlas (Desikan et al., 2006). This atlas was both used to localize significant VBM and SBM results (CAT), and for subject-specific parcellations which were rigidly coregistered in native space (ANTs) to the rsfMRI data, in order to construct a functional connectome. These subject-specific connectomes consisted of adapted Desikan-Killiany atlas nodes (the atlas was complemented with additional nodes of interest, see Figure, and node list below) and positive partial correlations between their average BOLD signals as their edge weights (in-house matlab scripts). 


\begin{tabular}{|c|c|}
\hline Left Hemisphere & Right Hemisphere \\
\hline 'Left-Cerebellum-Cortex' & 'Right-Cerebellum-Cortex' \\
\hline 'Left-Thalamus-Proper' & 'Right-Thalamus-Proper' \\
\hline 'Left-Caudate' & 'Right-Caudate' \\
\hline 'Left-Putamen' & 'Right-Putamen' \\
\hline 'Left-Pallidum' & 'Right-Pallidum' \\
\hline 'Left-Hippocampus' & 'Right-Hippocampus' \\
\hline 'Left-Amygdala' & 'Right-Amygdala' \\
\hline 'Left-Accumbens-area' & 'Right-Accumbens-area' \\
\hline 'Left-VentralDC' & 'Right-VentralDC' \\
\hline 'ctx-lh-caudalanteriorcingulate' & 'ctx-rh-caudalanteriorcingulate' \\
\hline 'ctx-lh-caudalmiddlefrontal' & 'ctx-rh-caudalmiddlefrontal' \\
\hline 'ctx-lh-cuneus' & 'ctx-rh-cuneus' \\
\hline 'ctx-lh-entorhinal' & 'ctx-rh-entorhinal' \\
\hline 'ctx-lh-fusiform' & 'ctx-rh-fusiform' \\
\hline 'ctx-lh-inferiorparietal' & 'ctx-rh-inferiorparietal' \\
\hline 'ctx-lh-inferiortemporal' & 'ctx-rh-inferiortemporal' \\
\hline 'ctx-lh-isthmuscingulate' & 'ctx-rh-isthmuscingulate' \\
\hline 'ctx-lh-lateraloccipital' & 'ctx-rh-lateraloccipital' \\
\hline 'ctx-lh-lateralorbitofrontal' & 'ctx-rh-lateralorbitofrontal' \\
\hline 'ctx-lh-lingual' & 'ctx-rh-lingual' \\
\hline 'ctx-lh-medialorbitofrontal' & 'ctx-rh-medialorbitofrontal' \\
\hline 'ctx-lh-middletemporal' & 'ctx-rh-middletemporal' \\
\hline 'ctx-lh-parahippocampal' & 'ctx-rh-parahippocampal' \\
\hline 'ctx-lh-paracentral' & 'ctx-rh-paracentral' \\
\hline 'ctx-lh-parsopercularis' & 'ctx-rh-parsopercularis' \\
\hline 'ctx-lh-parsorbitalis' & 'ctx-rh-parsorbitalis' \\
\hline 'ctx-lh-parstriangularis' & 'ctx-rh-parstriangularis' \\
\hline 'ctx-lh-pericalcarine' & 'ctx-rh-pericalcarine' \\
\hline 'ctx-lh-postcentral' & 'ctx-rh-postcentral' \\
\hline 'ctx-lh-posteriorcingulate' & 'ctx-rh-posteriorcingulate' \\
\hline 'ctx-lh-precentral' & 'ctx-rh-precentral' \\
\hline 'ctx-lh-precuneus' & 'ctx-rh-precuneus' \\
\hline 'ctx-lh-rostralanteriorcingulate' & 'ctx-rh-rostralanteriorcingulate' \\
\hline 'ctx-lh-rostralmiddlefrontal' & 'ctx-rh-rostralmiddlefrontal' \\
\hline 'ctx-lh-superiorfrontal' & 'ctx-rh-superiorfrontal' \\
\hline 'ctx-lh-superiorparietal' & 'ctx-rh-superiorparietal' \\
\hline 'ctx-lh-superiortemporal' & 'ctx-rh-superiortemporal' \\
\hline 'ctx-lh-supramarginal' & 'ctx-rh-supramarginal' \\
\hline 'ctx-lh-frontalpole' & 'ctx-rh-frontalpole' \\
\hline
\end{tabular}


517

518

519

520
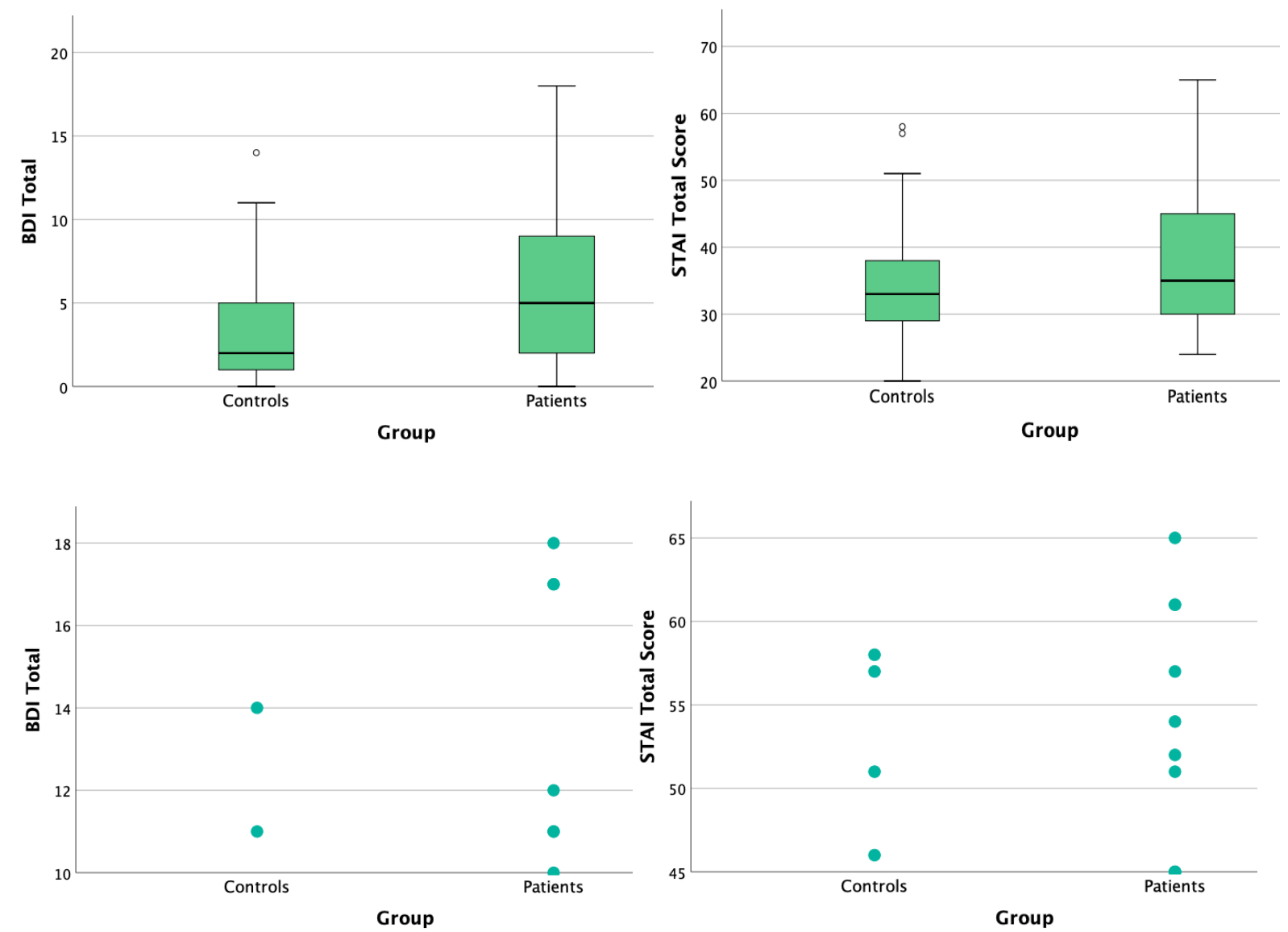

521

522

523

Independent samples T-test

\begin{tabular}{llllll}
\hline Group BDI & Mean & SD & SE & T-statistic & p-value \\
\hline Control & 3.68 & 3.488 & .598 & -2.365 & .021 \\
Patient & 6.16 & 4.946 & .874 & & \\
\hline Group STAI & & & & & .149 \\
\hline Control & 34.65 & 8.927 & 1.531 & -1.460 & \\
Patient & 38.41 & 11.870 & 2.098 & & \\
\hline
\end{tabular}

524

525 Correlations with left hemispheric cortical thickness

\begin{tabular}{rrrr}
\hline & RMFC & LOFC & parahipp \\
\hline BDI & -.001 & -.093 & -.239 \\
$p$ & .993 & .462 & .055 \\
\hline & & & \\
\hline STAI & -.005 & -.007 & -.071 \\
$p$ & .971 & .957 & .573 \\
\hline
\end{tabular}




\subsection{Post-hoc nodal strength analyses of all ROIs}

527

528

529

530

531

532

533

534

535

536

537

538

539

540

541

542

543

544

545

546

547

In order to assess specific vulnerability of the parahippocampal region, the prevalence of significant group differences in nodal strength was explored across all brain regions, with additional post-hoc group comparisons of nodal strength of all nodes (including the remaining 80 ROIs, cost-integrated over 33\% network density), non-corrected for multiple comparisons. This additional exploration yielded only 7 regions with different nodal strength values between the two groups, including the left parahippocampal region. This suggests that group differences in functional coherence do not appear widespread across the brain, but are limited to certain areas including the parahippocampal area specifically.

\subsection{Post-hoc analyses for SES effects}

\section{1) SES main effect analyses} VBM:

With regard to the VBM analysis, a main effect of SES was only encountered at uncorrected p-value of 0.001 (see Figure below). Three focused clusters of max. 6voxels appeared significant at uncorrected level. However, after FDR-correction, SES does not show any significant main effects. Furthermore, the three clusters at uncorrected level, do not overlap with the group differences, so this does not seem to be related with the significant group differences at FDR-correction level.

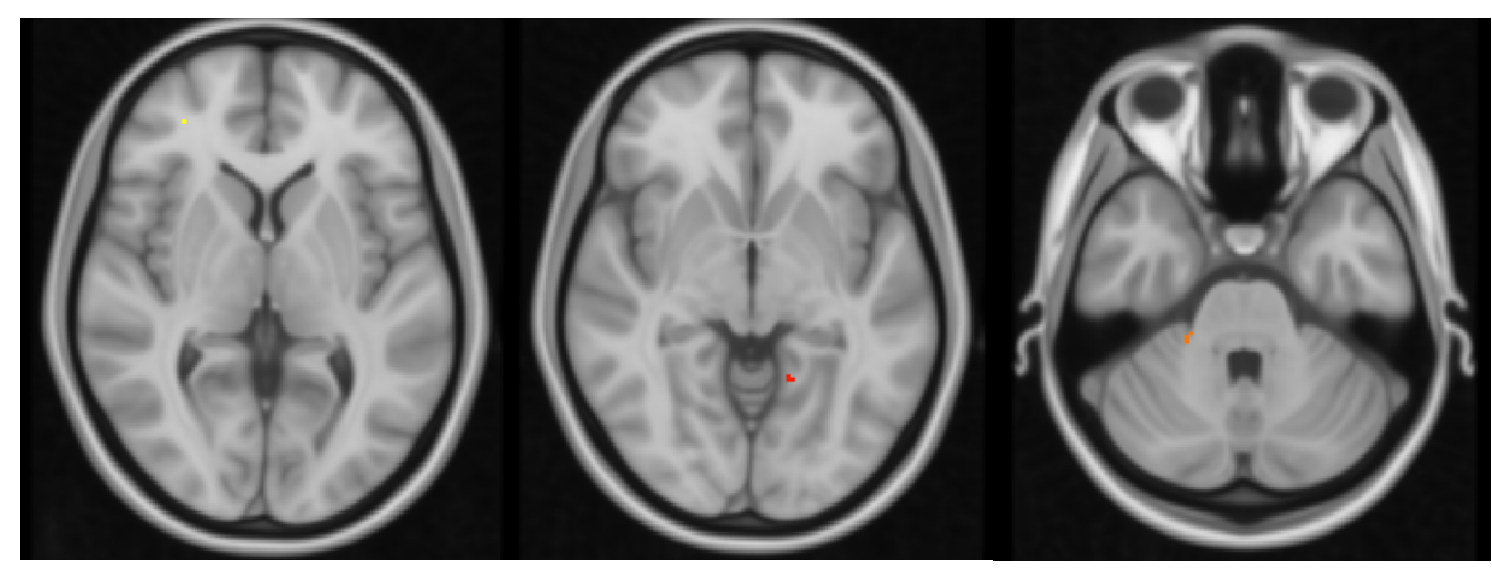


549 No significant main effects of SES on cortical thickness were encountered across the entire

550 group. In addition, in patients only, SES did not appear as an additional risk factor for cortical

551 thickness:

\begin{tabular}{|c|c|c|}
\hline & & SES \\
\hline \multirow[t]{2}{*}{ cortthickness_lh_rostralmiddlefrontal } & Pearson Correlation &,- 021 \\
\hline & Sig. (2-tailed) & ,908 \\
\hline \multirow[t]{2}{*}{ cortthickness_rh_rostralmiddlefrontal } & Pearson Correlation &,- 018 \\
\hline & Sig. (2-tailed) & ,922 \\
\hline \multirow[t]{2}{*}{ cortthickness_rh_lateralorbitofrontal } & Pearson Correlation &,- 286 \\
\hline & Sig. (2-tailed) &, 112 \\
\hline \multirow[t]{2}{*}{ cortthickness_lh_parahipp_40DK } & Pearson Correlation & ,094 \\
\hline & Sig. (2-tailed) &, 608 \\
\hline
\end{tabular}

552

553

554

555

556

557

558

559

560 areas).

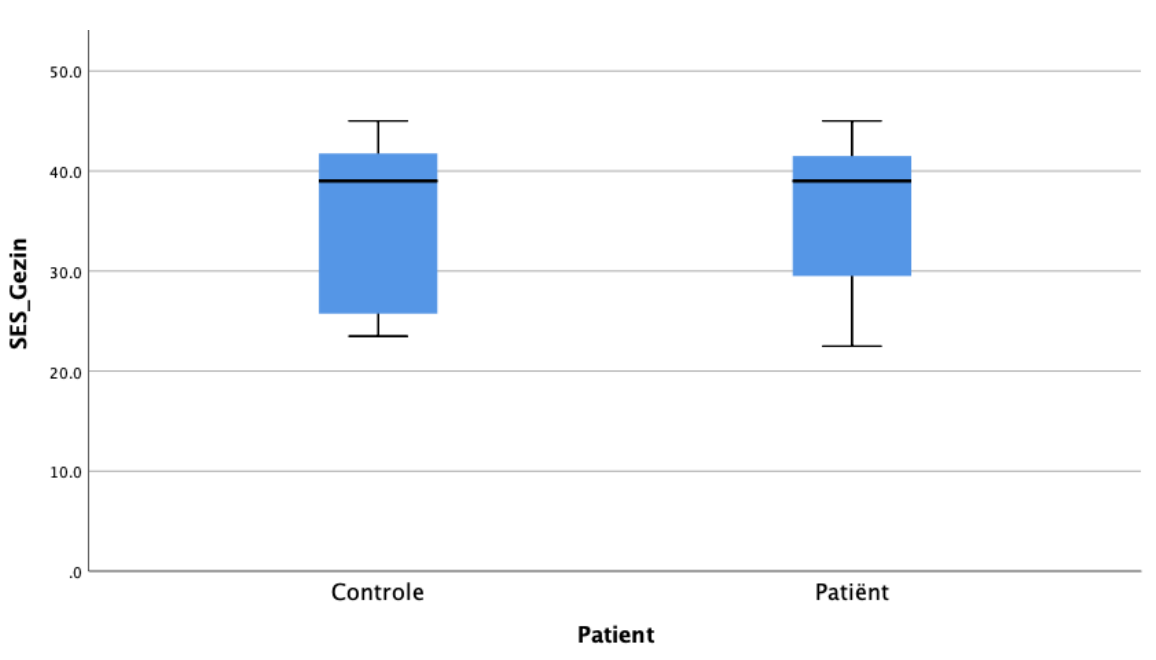

2) Group comparison patients vs controls after SES outlier exclusion $\underline{\mathrm{VBM}}$

Data of patients and controls with SES scores between 22 and 48 were extracted for subanalyses. These subgroups of these Patients $(n=22)$ versus Controls $(n=9)$ were more closely matched for their SES scores (see Boxplots below) and did not significantly differ in their SES scores $(\mathrm{T}=-.087, \mathrm{p}=.931)$, but still show significant group differences in grey matter volumes, which are similar to our original analyses (including the orbitofrontal and cerebellar 
563 In this sub-analysis, the regions with larger grey matter volumes in controls are depicted in

564 red:

565

566

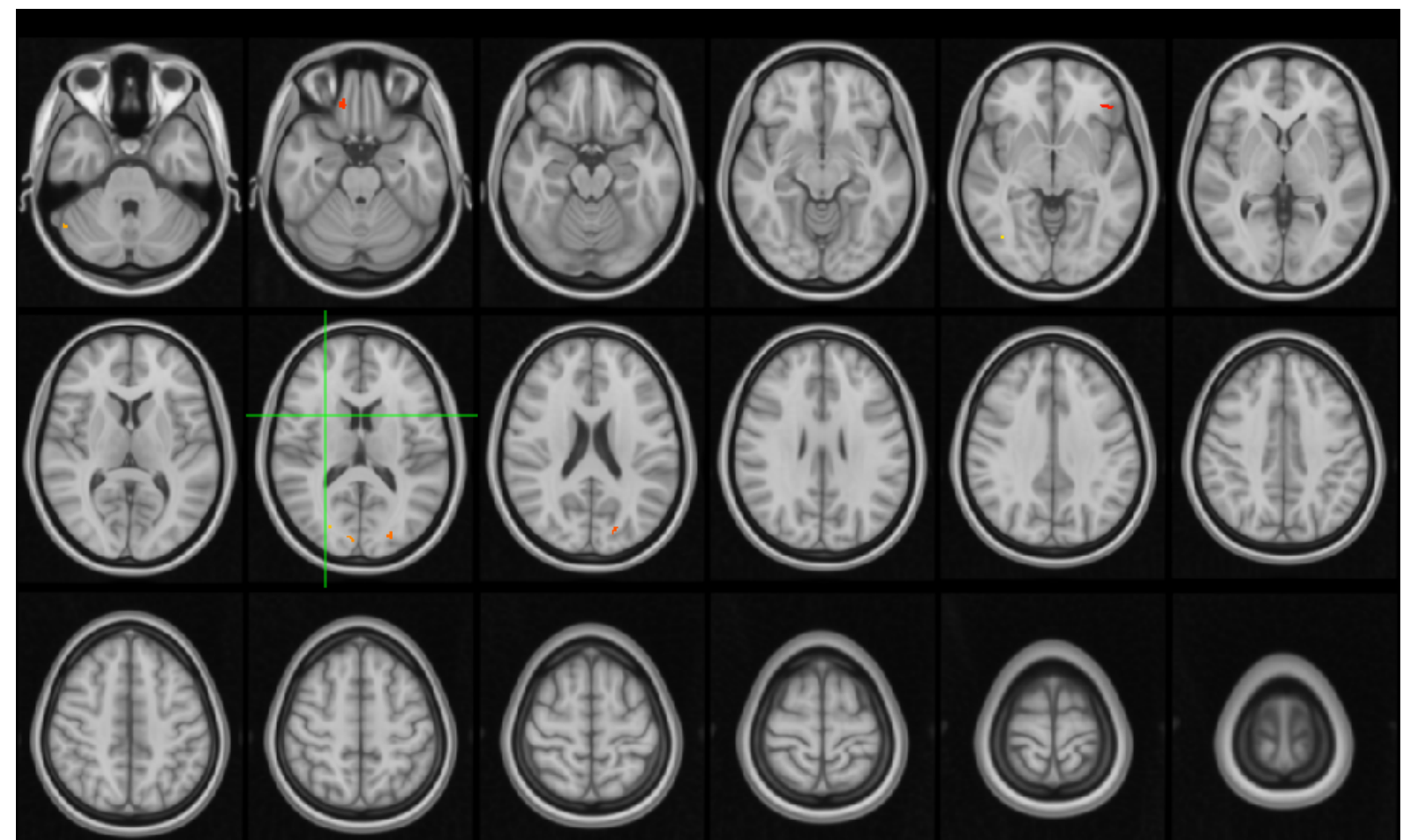

567

$568 \underline{\text { SBM }}$

569 With regard to the SBM analyses in the selected subgroups with matched SES scores, these

570 analyses again confirmed smaller cortical thickness in patients compared to controls (patients

$571<$ controls) (see Figure below). Again, no significant differences in cortical thickness were

572 encountered in the opposite direction (i.e. cortical thickness patients $>$ controls). The regions

573 that appeared significant included the right temporal pole, pre- and postcentral regions, the

574 left postcentral, superior parietal, rostral medial frontal, superior frontal, temporal pole,

575 posterior cingulate. Of these regions, the left rostral medial frontal region was replicated from

576 the initial (whole group analyses), which strengthens our confidence in these findings. 
577
578
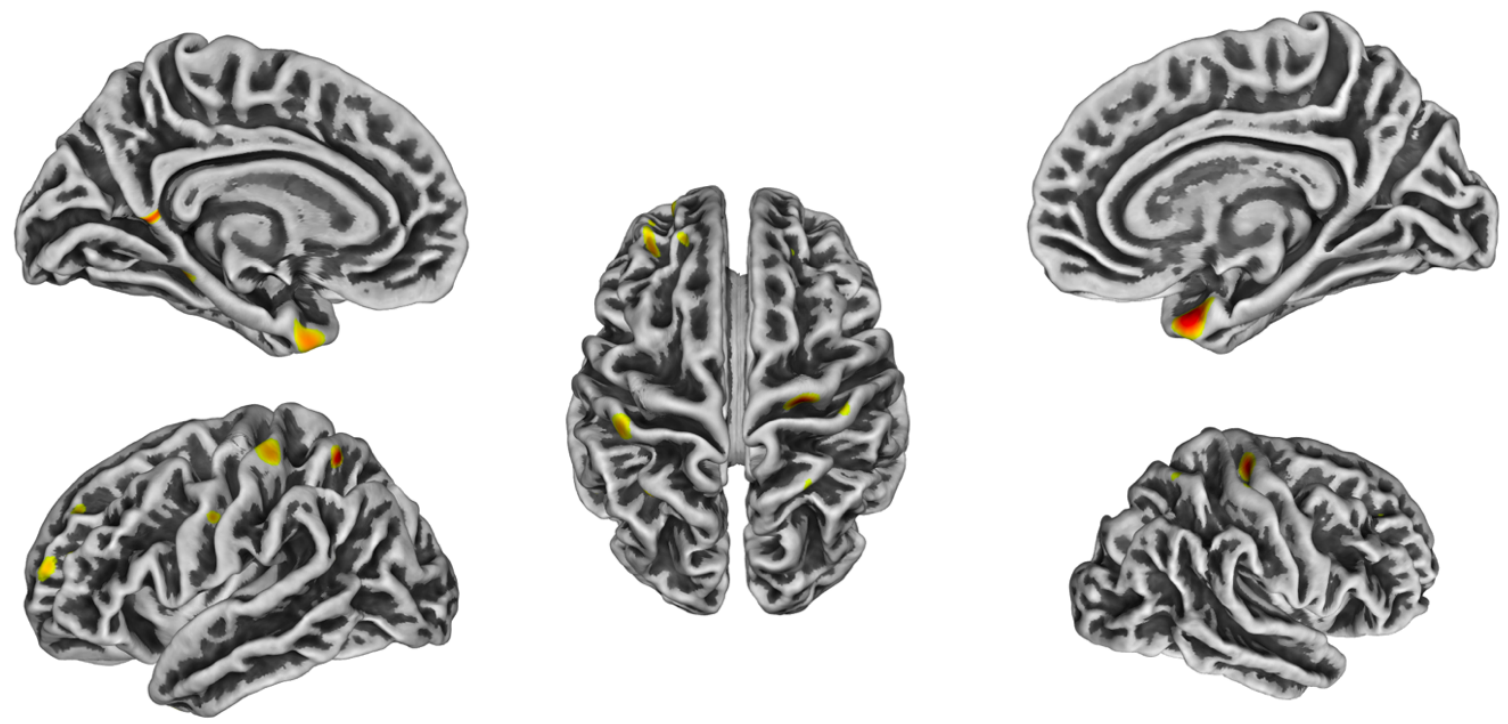


\section{References}

Achard, S., Bullmore, E., 2007. Efficiency and cost of economical brain functional networks. PLoS Comput. Biol. https://doi.org/10.1371/journal.pcbi.0030017

Aggleton, J.P., 2012. Multiple anatomical systems embedded within the primate medial temporal lobe: Implications for hippocampal function. Neurosci. Biobehav. Rev. https://doi.org/10.1016/j.neubiorev.2011.09.005

Ahles, T., Saykin, A., 2007. Candidate mechanisms for chemotherapy-induced cognitive changes. Nat. Rev. Cancer 7, 192-201. https://doi.org/10.1038/nrc2073

Amidi, A., Agerbaek, M., Wu, L.M., Pedersen, A.D., Mehlsen, M., Clausen, C.R., Demontis, D., Borglum, A.D., Harboll, A., Zachariae, R., 2016. Changes in cognitive functions and cerebral grey matter and their associations with inflammatory markers, endocrine markers, and APOE genotypes in testicular cancer patients undergoing treatment. Brain Imaging Behav. 1-15. https://doi.org/10.1007/s11682-016-9552-3

Amidi, A., Hosseini, S.M.H., Leemans, A., Kesler, S.R., Agerbæk, M., Wu, L.M., Zachariae, R., 2017. Changes in Brain Structural Networks and Cognitive Functions in Testicular Cancer Patients Receiving Cisplatin-Based Chemotherapy. J. Natl. Cancer Inst. 109. https://doi.org/10.1093/jnci/djx085

Beck, A.T., Steer, R.A., Carbin, M.G., 1988. Psychometric properties of the Beck Depression Inventory: Twenty-five years of evaluation. Clin. Psychol. Rev. https://doi.org/10.1016/0272-7358(88)90050-5

Billiet*, T., Elens*, I., Sleurs*, C., Uyttebroeck, A., D’Hooge, R., Lemiere, J., Deprez, S., 2018. Brain Connectivity and Cognitive Flexibility in Nonirradiated Adult Survivors of Childhood Leukemia. JNCI J. Natl. Cancer Inst. 110, 1-9. https://doi.org/10.1093/jnci/djy009

Blommaert, J., Schroyen, G., Vandenbulcke, M., Radwan, A., Smeets, A., Peeters, R., Sleurs, C., Neven, P., Wildiers, H., Amant, F., Sunaert, S., Deprez, S., 2019. Age-dependent 
brain volume and neuropsychological changes after chemotherapy in breast cancer patients. Hum. Brain Mapp. Epub.

607

608

609

610

611

612

613

614

615

616

617

618

619

620

621

622

623

624

625

626

627

628

629

Conroy, S.K., McDonald, B.C., Smith, D.J., Moser, L.R., West, J.D., Kamendulis, L.M., Klaunig, J.E., Champion, V.L., Unverzagt, F.W., Saykin, A.J., 2013. Alterations in brain structure and function in breast cancer survivors: effect of post-chemotherapy interval and relation to oxidative DNA damage. Breast Cancer Res. Treat. 137, 493-502. https://doi.org/10.1007/s10549-012-2385-x

Correa, D.D., Root, J.C., Kryza-Lacombe, M., Mehta, M., Karimi, S., Hensley, M.L., Relkin, N., 2016. Brain structure and function in patients with ovarian cancer treated with firstline chemotherapy: a pilot study. Brain Imaging Behav. 1-12. https://doi.org/10.1007/s11682-016-9608-4

D’Agostino, N.M., Edelstein, K., Zhang, N., Recklitis, C.J., Brinkman, T.M., Srivastava, D., Leisenring, W.M., Robison, L.L., Armstrong, G.T., Krull, K.R., 2016. Comorbid symptoms of emotional distress in adult survivors of childhood cancer. Cancer 122, 3215-3224. https://doi.org/10.1002/cncr.30171

De Ruiter, M.B., Reneman, L., Boogerd, W., Veltman, D.J., Caan, M., Douaud, G., Lavini, C., Linn, S.C., Boven, E., Van Dam, F.S.A.M.A.M., Schagen, S.B., 2012. Late effects of high-dose adjuvant chemotherapy on white and gray matter in breast cancer survivors: Converging results from multimodal magnetic resonance imaging. Hum. Brain Mapp. 33, 2971-2983. https://doi.org/10.1002/hbm.21422

Deprez, S., Amant, F., Smeets, A., Peeters, R., Leemans, A., Van Hecke, W., Verhoeven, J.S., Christiaens, M.-R., Vandenberghe, J., Vandenbulcke, M., Sunaert, S., 2012. Longitudinal assessment of chemotherapy-induced structural changes in cerebral white matter and its correlation with impaired cognitive functioning. J. Clin. Oncol. 30, 274-281. https://doi.org/10.1200/JCO.2011.36.8571 
Desikan, R.S., Ségonne, F., Fischl, B., Quinn, B.T., Dickerson, B.C., Blacker, D., Buckner, R.L., Dale, A.M., Maguire, R.P., Hyman, B.T., Albert, M.S., Killiany, R.J., 2006. An automated labeling system for subdividing the human cerebral cortex on MRI scans into gyral based regions of interest. Neuroimage. https://doi.org/10.1016/j.neuroimage.2006.01.021

Duncan, N.W., Hayes, D.J., Wiebking, C., Tiret, B., Pietruska, K., Chen, D.Q., Rainville, P., Marjańska, M., Ayad, O., Doyon, J., Hodaie, M., Northoff, G., 2015. Negative childhood experiences alter a prefrontal-insular-motor cortical network in healthy adults: A preliminary multimodal rsfMRI-fMRI-MRS-dMRI study. Hum. Brain Mapp. 36, 46224637. https://doi.org/10.1002/hbm.22941

Edelmann, M.N., Daryani, V.M., Bishop, M.W., Liu, W., Brinkman, T.M., Stewart, C.F., Mulrooney, D.A., Kimberg, C., Ness, K.K., Cheung, Y.T., Srivastava, D.K., Robison, L.L., Hudson, M.M., Krull, K.R., 2016. Neurocognitive and Patient-Reported Outcomes in Adult Survivors of Childhood Osteosarcoma. JAMA Oncol. 2, 201-208. https://doi.org/10.1001/jamaoncol.2015.4398

Feng, Y., Zhang, X., Zheng, G., Zhang, L., 2019. Chemotherapy-induced brain changes in breast cancer survivors: evaluation with multimodality magnetic resonance imaging. Brain Imaging Behav. Epub.

Frederick, N.N., Kenney, L., Vrooman, L., Recklitis, C.J., 2016. Fatigue in adolescent and adult survivors of non-CNS childhood cancer: a report from project REACH. Support. Care Cancer 24, 3951-9. https://doi.org/10.1007/s00520-016-3230-2

Friend, A.J., Feltbower, R.G., Hughes, E.J., Dye, K.P., Glaser, A.W., 2018. Mental health of long-term survivors of childhood and young adult cancer: A systematic review. Int. J. Cancer. https://doi.org/10.1002/ijc.31337

Gaser, C., Dahnke, R., 2016. CAT - A Computational Anatomy Toolbox for the Analysis of 
Genschaft, M., Huebner, T., Plessow, F., Ikonomidou, V.N., Abolmaali, N., Krone, F.,

657

658

659

660

661

662

663

664

665

666

667

668

669

670

671

672

673

674

675

676

677

678

679

Hoffmann, A., Holfeld, E., Vorwerk, P., Kramm, C., Gruhn, B., Koustenis, E., Hernaiz-

Driever, P., Mandal, R., Suttorp, M., Hummel, T., Ikonomidou, C., Kirschbaum, C., Smolka, M.N., 2013. Impact of chemotherapy for childhood leukemia on brain morphology and function. PLoS One 8. https://doi.org/10.1371/journal.pone.0078599

Giedd, J.N., Blumenthal, J., Jeffries, N.O., Castellanos, F.X., Liu, H., Zijdenbos, A., Paus, T., Evans, A.C., Rapoport, J.L., 1999. Brain development during childhood and adolescence: a longitudinal MRI study. Nat. Neurosci. 2, 861-863. https://doi.org/10.1038/13158

Hakamata, Y., Matsuoka, Y., Inagaki, M., Nagamine, M., Hara, E., Imoto, S., Murakami, K., Kim, Y., Uchitomi, Y., 2007. Structure of orbitofrontal cortex and its longitudinal course in cancer-related post-traumatic stress disorder. Neurosci. Res. 59, 383-389. https://doi.org/10.1016/j.neures.2007.08.012

Hampson, J.P., Zick, S.M., Khabir, T., Wright, B.D., Harris, R.E., 2015. Altered resting brain connectivity in persistent cancer related fatigue. NeuroImage. Clin. 8, 305-313. https://doi.org/10.1016/j.nicl.2015.04.022

Ikonomidou, C., 2018. Chemotherapy and the pediatric brain. Mol. Cell. Pediatr. https://doi.org/10.1186/s40348-018-0087-0

Jaworska, N., Yücel, K., Courtright, A., Macmaster, F.P., Sembo, M., Macqueen, G., 2016. Subgenual anterior cingulate cortex and hippocampal volumes in depressed youth: The role of comorbidity and age. J. Affect. Disord. https://doi.org/10.1016/j.jad.2015.10.064 Jenkinson, M., Pechaud, M., Smith, S., 2002. BET2 - MR-Based Estimation of Brain, Skull and Scalp Surfaces. Elev. Annu. Meet. Organ. Hum. Brain Mapp. 17, 2002. https://doi.org/citeulike-article-id:1179617 
Kadan-Lottick, N.S., Zeltzer, L.K., Liu, Q., Yasui, Y., Ellenberg, L., Gioia, G., Robison, L.L., Krull, K.R., 2010. Neurocognitive functioning in adult survivors of childhood noncentral nervous system cancers. J. Natl. Cancer Inst. 102, 881-893. https://doi.org/10.1093/jnci/djq156

Kaiser, J., Bledowski, C., Dietrich, J.J., 2014. Neural correlates of chemotherapy-related cognitive impairment. Cortex. 54, 33-50. https://doi.org/10.1016/j.cortex.2014.01.010

Kesler, S.R., Adams, M., Packer, M., Rao, V., Henneghan, A.M., Blayney, D.W., Palesh, O., 2017. Disrupted brain network functional dynamics and hyper-correlation of structural and functional connectome topology in patients with breast cancer prior to treatment. Brain Behav. 7, e00643. https://doi.org/10.1002/brb3.643

Kesler, S.R., Bennett, F.C., Mahaffey, M.L., Spiegel, D., 2009. Regional brain activation during verbal declarative memory in metastatic breast cancer. Clin. Cancer Res. 15, 6665-73. https://doi.org/10.1158/1078-0432.CCR-09-1227

Kesler, S.R., Gugel, M., Pritchard-Berman, M., Lee, C., Kutner, E., Hosseini, S.M.H., Dahl, G., Lacayo, N., 2014. Altered resting state functional connectivity in young survivors of acute lymphoblastic leukemia. Pediatr. Blood Cancer 61, 1295-1299. https://doi.org/10.1002/pbc.25022

Kharitonova, M., Martin, R.E., Gabrieli, J.D.E., Sheridan, M.A., 2013. Cortical gray-matter thinning is associated with age-related improvements on executive function tasks. Dev. Cogn. Neurosci. 6, 61-71. https://doi.org/10.1016/j.den.2013.07.002

Ki Moore, I.M., Hockenberry, M.J., Krull, K.R., 2013. Cancer-related cognitive changes in children, adolescents and adult survivors of childhood cancers. Semin. Oncol. Nurs. 29, 248-259. https://doi.org/10.1016/j.soncn.2013.08.005

Koppelmans, V., De Ruiter, M.B., Van Der Lijn, F., Boogerd, W., Seynaeve, C., Van Der Lugt, A., Vrooman, H., Niessen, W.J., Breteler, M.M.B., Schagen, S.B., 2012. Global 
and focal brain volume in long-term breast cancer survivors exposed to adjuvant chemotherapy. Breast Cancer Res. Treat. 132, 1099-1106.

707 https://doi.org/10.1007/s10549-011-1888-1

708

709

710

711

712

713

714

715

716

717

718

719

720

721

722

723

Krull, K.R., Cheung, Y.T., Liu, W., Fellah, S., Reddick, W.E., Brinkman, T.M., Kimberg, C., Ogg, R., Srivastava, D., Pui, C.-H., Robison, L.L., Hudson, M.M., 2016. Chemotherapy Pharmacodynamics and Neuroimaging and Neurocognitive Outcomes in Long-Term Survivors of Childhood Acute Lymphoblastic Leukemia. www.jco.org June J Clin Oncol 6, 2644-2653. https://doi.org/10.1200/JCO.2015.65.4574

Lähteenmäki, P.M., Krause, C.M., Sillanmäki, L., Salmi, T.T., Lang, A.H., 1999. Eventrelated alpha synchronization/desynchronization in a memory-search task in adolescent survivors of childhood cancer. Clin. Neurophysiol. 110, 2064-2073. https://doi.org/10.1016/S1388-2457(99)00170-4

Lepage, C., Smith, A.M., Moreau, J., Barlow-Krelina, E., Wallis, N., Collins, B., MacKenzie, J., Scherling, C., 2014. A prospective study of grey matter and cognitive function alterations in chemotherapy-treated breast cancer patients. Springerplus 3, 444. https://doi.org/10.1186/2193-1801-3-444

Li, X., Chen, H., Lv, Y., Chao, H.H., Gong, L., Li, C.S.R., Cheng, H., 2018. Diminished gray matter density mediates chemotherapy dosage-related cognitive impairment in breast cancer patients. Sci. Rep. https://doi.org/10.1038/s41598-018-32257-w

Marusak, H.A., Iadipaolo, A.S., Harper, F.W., Elrahal, F., Taub, J.W., Goldberg, E., Rabinak, C.A., 2017. Neurodevelopmental consequences of pediatric cancer and its treatment: applying an early adversity framework to understanding cognitive, behavioral, and emotional outcomes. Neuropsychol. Rev. 1-53. https://doi.org/10.1007/s11065-0179365-1

McDonald, B.C., Conroy, S.K., Ahles, T.A., West, J.D., Saykin, A.J., 2010. Gray matter 
reduction associated with systemic chemotherapy for breast cancer: a prospective MRI study. Breast Cancer Res. Treat. 123, 819-828. https://doi.org/10.1007/s10549-010$1088-4 ; 10.1007 / \mathrm{s} 10549-010-1088-4$

McDonald, B.C., Conroy, S.K., Smith, D.J., West, J.D., Saykin, A.J., 2013. Frontal gray matter reduction after breast cancer chemotherapy and association with executive symptoms: a replication and extension study. Brain. Behav. Immun. 30 Suppl, S117-25. https://doi.org/10.1016/j.bbi.2012.05.007

McDonald, B.C., Saykin, A.J., 2013. Alterations in brain structure related to breast cancer and its treatment: Chemotherapy and other considerations. Brain Imaging Behav. https://doi.org/10.1007/s11682-013-9256-x

Mohrmann, C., Henry, J., Hauff, M., Hayashi, R.J., 2015. Neurocognitive Outcomes and School Performance in Solid Tumor Cancer Survivors Lacking Therapy to the Central Nervous System. J. Pers. Med. 5, 83-90.

Murphy, K., Fox, M.D., 2017. Towards a consensus regarding global signal regression for resting state functional connectivity MRI. Neuroimage 154, 169-173. https://doi.org/10.1016/j.neuroimage.2016.11.052

Pohler, G., 1992. [An overview of studies on Simonton training in treatment of cancer patients]. Z. Arztl. Fortbild. (Jena). 86, 1109-1111.

Porto, L., Preibisch, C., Hattingen, E., Bartels, M., Lehrnbecher, T., Dewitz, R., Zanella, F., Good, C., Lanfermann, H., DuMesnil, R., Kieslich, M., 2008. Voxel-based morphometry and diffusion-tensor MR imaging of the brain in long-term survivors of childhood leukemia. Eur. Radiol. 18, 2691-2700. https://doi.org/10.1007/s00330-008-1038-2

Pruim, R.H.R., Mennes, M., van Rooij, D., Llera, A., Buitelaar, J.K., Beckmann, C.F., 2015. ICA-AROMA: A robust ICA-based strategy for removing motion artifacts from fMRI data. Neuroimage. https://doi.org/10.1016/j.neuroimage.2015.02.064 
Reddick, W.E., Glass, J.O., Johnson, D.P., Laningham, F.H., Pui, C.H., 2009. Voxel-based analysis of T2 hyperintensities in white matter during treatment of childhood leukemia. Am. J. Neuroradiol. 30, 1947-1954. https://doi.org/10.3174/ajnr.A1733

Reuter-Lorenz, P.A., Cimprich, B., 2013. Cognitive function and breast cancer: promise and potential insights from functional brain imaging. Breast Cancer Res. Treat. 137, 33-43. https://doi.org/10.1007/s10549-012-2266-3

Rock, P.L., Roiser, J.P., Riedel, W.J., Blackwell, A.D., 2014. Cognitive impairment in depression: a systematic review and meta-analysis. Psychol. Med. 44, 2029-40. https://doi.org/10.1017/S0033291713002535

Rothschild, G., Eban, E., Frank, L.M., 2017. A cortical-hippocampal-cortical loop of information processing during memory consolidation. Nat. Neurosci. 20, 251-259. https://doi.org/10.1038/nn.4457

Rubinov, M., Sporns, O., 2010. Complex network measures of brain connectivity: Uses and interpretations. Neuroimage. https://doi.org/10.1016/j.neuroimage.2009.10.003

Rzeski, W., Pruskil, S., Macke, A., Felderhoff-Mueser, U., Reiher, A.K., Hoerster, F., Jansma, C., Jarosz, B., Stefovska, V., Bittigau, P., Ikonomidou, C., 2004. Anticancers agents are potent neurotoxins in vitro and in vivo. Ann. Neurol. 56, 351-360. https://doi.org/10.1002/ana.20185

Sapolsky, R.M., 2001. Atrophy of the hippocampus in posttraumatic stress disorder: How and when? Hippocampus 11, 90-91. https://doi.org/10.1002/hipo.1026

Schmaal, L., Hibar, D.P., Sämann, P.G., Hall, G.B., Baune, B.T., Jahanshad, N., Cheung, J.W., Van Erp, T.G.M., Bos, D., Ikram, M.A., Vernooij, M.W., Niessen, W.J., Tiemeier, H., Hofman, A., Wittfeld, K., Grabe, H.J., Janowitz, D., Bülow, R., Selonke, M., Völzke, H., Grotegerd, D., Dannlowski, U., Arolt, V., Opel, N., Heindel, W., Kugel, H., Hoehn, D., Czisch, M., Couvy-Duchesne, B., Rentería, M.E., Strike, L.T., Wright, M.J., Mills, 

Goya-Maldonado, R., Gruber, O., Krämer, B., Hatton, S.N., Lagopoulos, J., Hickie, I.B., Frodl, T., Carballedo, A., Frey, E.M., Van Velzen, L.S., Penninx, B.W.J.H., Van Tol, M.J., Van der Wee, N.J., Davey, C.G., Harrison, B.J., Mwangi, B., Cao, B., Soares, J.C., Veer, I.M., Walter, H., Schoepf, D., Zurowski, B., Konrad, C., Schramm, E., Normann, C., Schnell, K., Sacchet, M.D., Gotlib, I.H., MacQueen, G.M., Godlewska, B.R., Nickson, T., McIntosh, A.M., Papmeyer, M., Whalley, H.C., Hall, J., Sussmann, J.E., Li, M., Walter, M., Aftanas, L., Brack, I., Bokhan, N.A., Thompson, P.M., Veltman, D.J., 2017. Cortical abnormalities in adults and adolescents with major depression based on brain scans from 20 cohorts worldwide in the ENIGMA Major Depressive Disorder

Seigers, R., Schagen, S.B., Beerling, W., Boogerd, W., van Tellingen, O., van Dam, cell proliferation and impaired cognitive performance by methotrexate in the rat. Behav. Brain Res. 186, 168-175. https://doi.org/10.1016/j.bbr.2007.08.004

Simo, M., Rifa-Ros, X., Rodriguez-Fornells, A., Bruna, J., 2013. Chemobrain: a systematic review of structural and functional neuroimaging studies. Neurosci. Biobehav. Rev. 37, 1311-1321. https://doi.org/10.1016/j.neubiorev.2013.04.015

Sleurs, C., Billiet, T., Peeters, R., Sunaert, S., Uyttebroeck, A., Deprez, S., 2018. Advanced MR diffusion imaging and chemotherapy-related changes in cerebral white matter microstructure of survivors of childhood bone and soft tissue sarcoma ? 1-13. Deprez, S., Uyttebroeck, A., 2019. Long-term leukoencephalopathy and neurocognitive functioning in childhood sarcoma patients treated with high-dose intravenous 
Sugimoto, S., Yamamoto, Y.L., Nagahiro, S., Diksic, M., 1995. Permeability change and brain tissue damage after intracarotid administration of cisplatin studied by double-tracer autoradiography in rats. J. Neurooncol. 24, 229-240.

Tamnes, C.K., Zeller, B., Amlien, I.K., Kanellopoulos, A., Andersson, S., Due-Tonnessen, P., Ruud, E., Walhovd, K.B., Fjell, A.M., 2015. Cortical surface area and thickness in adult survivors of pediatric acute lymphoblastic leukemia. Pediatr. Blood Cancer 62, $1027-$ 1034. https://doi.org/10.1002/pbc. 25386

Thompson, P.M., Stein, J.L., Medland, S.E., Hibar, D.P., Vasquez, A.A., Renteria, M.E., Toro, R., Jahanshad, N., Schumann, G., Franke, B., Wright, M.J., Martin, N.G., Agartz, I., Alda, M., Alhusaini, S., Almasy, L., Almeida, J., Alpert, K., Andreasen, N.C., Andreassen, O.A., Apostolova, L.G., Appel, K., Armstrong, N.J., Aribisala, B., Bastin, M.E., Bauer, M., Bearden, C.E., Bergmann, Ø., Binder, E.B., Blangero, J., Bockholt, R.M., Brunner, H.G., Brohawn, D.G., Buckner, R.L., Buitelaar, J., Bulayeva, K., Bustillo, J.R., Calhoun, V.D., Cannon, D.M., Cantor, R.M., Carless, M.A., Caseras, X., Cavalleri, G.L., Chakravarty, M.M., Chang, K.D., Ching, C.R.K., Christoforou, A., Cichon, S., Clark, V.P., Conrod, P., Coppola, G., Crespo-Facorro, B., Curran, J.E., Czisch, M., Deary, I.J., de Geus, E.J.C., den Braber, A., Delvecchio, G., Depondt, C., de Haan, L., de Zubicaray, G.I., Dima, D., Dimitrova, R., Djurovic, S., Dong, H., Donohoe, G., Duggirala, R., Dyer, T.D., Ehrlich, S., Ekman, C.J., Elvsåshagen, T., Emsell, L., Erk, S., Espeseth, T., Fagerness, J., Fears, S., Fedko, I., Fernández, G., Fisher, S.E., Foroud, T., Fox, P.T., Francks, C., Frangou, S., Frey, E.M., Frodl, T., Frouin, V., Garavan, H., Giddaluru, S., Glahn, D.C., Godlewska, B., Goldstein, R.Z., Gollub, R.L., Grabe, H.J., 
A., Toga, A.W., Tordesillas-Gutierrez, D., Trabzuni, D., Trost, S., Turner, J., Van den Heuvel, M., van der Wee, N.J., van Eijk, K., van Erp, T.G.M., van Haren, N.E.M., van 't Ent, D., van Tol, M.J., Valdés Hernández, M.C., Veltman, D.J., Versace, A., Völzke, H., Walker, R., Walter, H., Wang, L., Wardlaw, J.M., Weale, M.E., Weiner, M.W., Wen, W., Westlye, L.T., Whalley, H.C., Whelan, C.D., White, T., Winkler, A.M., Wittfeld, K., Woldehawariat, G., Wolf, C., Zilles, D., Zwiers, M.P., Thalamuthu, A., Schofield, P.R., Freimer, N.B., Lawrence, N.S., Drevets, W., 2014. The ENIGMA Consortium: Largescale collaborative analyses of neuroimaging and genetic data. Brain Imaging Behav. 8, 153-182. https://doi.org/10.1007/s11682-013-9269-5

Tustison, N.J., Avants, B.B., Cook, P.A., Zheng, Y., Egan, A., Yushkevich, P.A., Gee, J.C., 2010. N4ITK: Improved N3 bias correction. IEEE Trans. Med. Imaging 29, 1310-1320. https://doi.org/10.1109/TMI.2010.2046908

White, T., Su, S., Schmidt, M., Kao, C.Y., Sapiro, G., 2010. The development of gyrification in childhood and adolescence. Brain Cogn. https://doi.org/10.1016/j.bandc.2009.10.009

Wick, A., Wick, W., Hirrlinger, J., Gerhardt, E., Dringen, R., Dichgans, J., Weller, M., Schulz, J.B., 2004. Chemotherapy-induced cell death in primary cerebellar granule neurons but not in astrocytes: In vitro paradigm of differential neurotoxicity. J. Neurochem. 91, 1067-1074. https://doi.org/10.1111/j.1471-4159.2004.02774.x

Willard, V.W., Cox, L.E., Russell, K.M., Kenney, A., Jurbergs, N., Molnar, A.E., Harman, J.L., 2017. Cognitive and Psychosocial Functioning of Preschool-Aged Children with Cancer. J. Dev. Behav. Pediatr. 38, 638-645. https://doi.org/10.1097/DBP.0000000000000512

Wilson, J.Z., Marin, D., Maxwell, K., Cumming, J., Berger, R., Saini, S., Ferguson, W., Chibnall, J.T., 2016. Association of Posttraumatic Growth and Illness-Related Burden With Psychosocial Factors of Patient, Family, and Provider in Pediatric Cancer 
Yi, J., Zebrack, B., Kim, M.A., Cousino, M., 2015. Posttraumatic Growth Outcomes and Their Correlates Among Young Adult Survivors of Childhood Cancer. J. Pediatr. Psychol. 40, 981-991. https://doi.org/10.1093/jpepsy/jsv075

Yoshikawa, E., Matsuoka, Y., Yamasue, H., Inagaki, M., Nakano, T., Akechi, T., Kobayakawa, M., Fujimori, M., Nakaya, N., Akizuki, N., Imoto, S., Murakami, K., Kasai, K., Uchitomi, Y., 2006. Prefrontal cortex and amygdala volume in first minor or major depressive episode after cancer diagnosis. Biol. Psychiatry 59, 707-712. https://doi.org/10.1016/j.biopsych.2005.08.018

Zhang, Y., Zou, P., Mulhern, R.K., Butler, R.W., Laningham, F.H., Ogg, R.J., 2008. Brain structural abnormalities in survivors of pediatric posterior fossa brain tumors: A voxelbased morphometry study using free-form deformation. Neuroimage 42, 218-229. https://doi.org/10.1016/j.neuroimage.2008.04.181 alteration in survivors of acute lymphoblastic leukemia with chemotherapy treatment: A voxel-based morphometry and diffusion tensor imaging study. Brain Res. 1658, 68-72. https://doi.org/10.1016/j.brainres.2017.01.017

897 